Accepted Manuscript

GAIA-SMAA-PROMETHEE for a hierarchy of interacting criteria

Sally Giuseppe Arcidiacono, Salvatore Corrente, Salvatore Greco

PII:

S0377-2217(18)30267-4

DOI:

10.1016/j.ejor.2018.03.038

Reference: $\quad$ EOR 15058

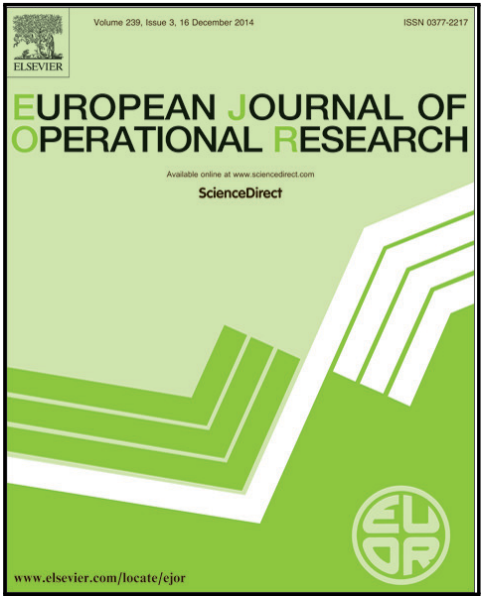

To appear in: $\quad$ European Journal of Operational Research

Received date: $\quad 15$ March 2017

Revised date: $\quad 23$ March 2018

Accepted date: $\quad 27$ March 2018

Please cite this article as: Sally Giuseppe Arcidiacono, Salvatore Corrente, Salvatore Greco, GAIASMAA-PROMETHEE for a hierarchy of interacting criteria, European Journal of Operational Research (2018), doi: 10.1016/j.ejor.2018.03.038

This is a PDF file of an unedited manuscript that has been accepted for publication. As a service to our customers we are providing this early version of the manuscript. The manuscript will undergo copyediting, typesetting, and review of the resulting proof before it is published in its final form. Please note that during the production process errors may be discovered which could affect the content, and all legal disclaimers that apply to the journal pertain. 


\section{Highlights}

- We propose an extension of the bipolar PROMETHEE methods

- The methodology handles problems with hierarchically structured criteria

- We extend the GAIA methodology to deal with such problems

- Robust ordinal regression and stochastic analysis deal with robustness issues

- We apply the proposed method to the ranking of pharmaceutical companies

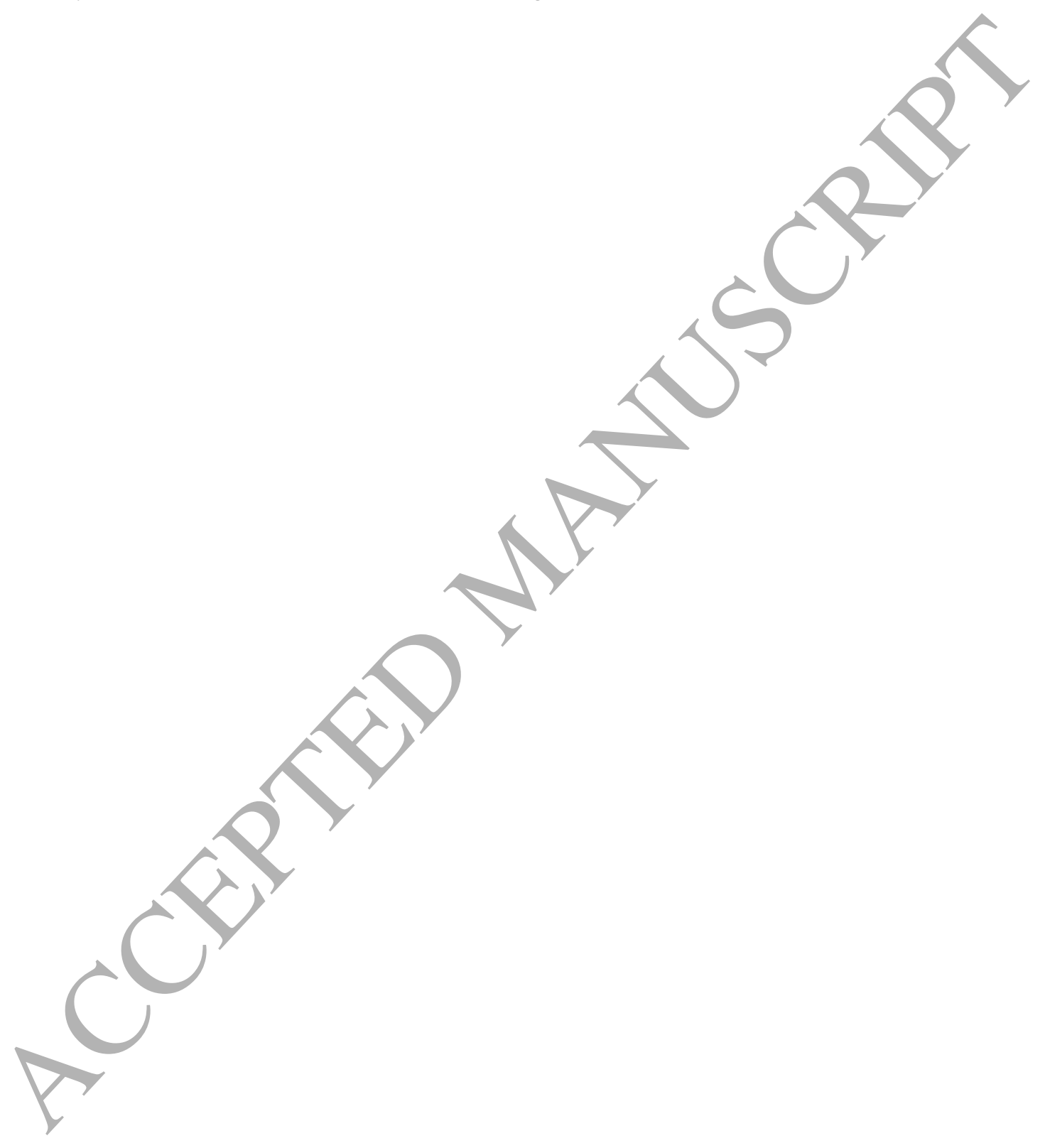




\title{
GAIA-SMAA-PROMETHEE for a hierarchy of interacting criteria
}

\author{
Sally Giuseppe Arcidiacono ${ }^{\mathrm{a}}$, Salvatore Corrente $^{\mathrm{a}}$, Salvatore Greco ${ }^{\mathrm{a}, \mathrm{b}}$ \\ ${ }^{a}$ Department of Economics and Business, University of Catania, Corso Italia, 55, 95129 Catania, Italy \\ ${ }^{b}$ University of Portsmouth, Portsmouth Business School, Centre of Operations Research and Logistics (CORL), \\ Richmond Building, Portland Street, Portsmouth PO1 3DE, United Kingdom
}

\begin{abstract}
In this paper we propose an extension of the PROMETHEE methods. Despite PROMETHEE are among the most applied methods in Multiple Criteria Decision Aiding (MCDA), some drawbacks can be underlined with respect to their applicability since they do not take into account few characteristics of multicriteria decision problems that are very relevant in real world applications: (i) robustness regarding the plurality of compatible preference parameters; (ii) interaction between criteria; (iii) hierarchies of criteria. Even if different extensions of the PROMETHEE methods have taken into account all these aspects singularly, we propose to deal with all of them simultaneously presenting a new version which incorporates Robust Ordinál Regression (ROR), Stochastic Multicriteria Acceptability Analysis (SMAA), bipolar Choquet integral and Multiple Criteria Hierarchy Process (MCHP). ROR and SMAA permit to consider all the instances of the considered preference model compatible with the preference information provided by the DM; the bipolar Choquet integral is able to represent the possible positive and negative interactions between criteria as well as the antagonistic effect between some of them; finally, the MCHP permits to decompose the problem in small parts so that each of them can be analyzed more in detail with respect to the problem at hand. Moreover, we also introduce an extension of the GAIA technique to handle visualization in MCDA problems presenting interactions and antagonistic effects between criteria organized in a hierarchy. Moreover, it gives the possibility to display the plurality of instances for the preference model considered by SMAA. A didactic example will illustrate the proposed methodology.
\end{abstract}

Keywords: Decision Support Systems, PROMETHEE methods, Hierarchy of Criteria, Robustness Concerns, Bipolar Choquet integral, GAIA, SMAA

\section{Introduction}

In taking any decision, we have to compare a plurality of alternatives evaluated with respect to different points of view, technically called criteria [24]. When the decision problem is too complex to be handled by common sense and personal experience, some formal approach can be used. Many multiple criteria methods have been proposed with this aim and among them some of the most appreciated are PROMETHEE methods [9].

Basic PROMETHEE methods make the following assumptions:

Email addresses: s.arcidiacono@unict.it (Sally Giuseppe Arcidiacono), salvatore.corrente@unict.it (Salvatore Corrente), salgreco@unict.it (Salvatore Greco) 
- there is a finite set of criteria: in general the considered criteria are not too numerous; moreover, it is also supposed that the criteria are not hierarchically organized;

- the criteria are not interacting: there are no phenomena of mutual strengthening or weakening, or antagonistic effects between them;

- it is possible to define precisely the parameters of the decision model: weights of considered criteria are supposed to be clearly fixed.

Real world decision problems are very often violating the above assumptions, so that the application of PROMETHEE methods in these cases is affected by a simplified reformulation which neglects several aspects that can be indeed quite salient for the definition of the final recommendation. For example, in risk-return evaluation of industrial companies, it is quite natural to cope with the following situations:

- taking into account economic indices such as:

- debt ratio, current ratio and quick ratio defining solvency and liquidity aspects,

- return on equity, return on investment and gross profit ratio defining the profitability aspects,

- interest expense ratio, account receivable ratio and administration cost per unit defining managerial aspects.

In these cases, it is therefore natural to compare industrial companies not only at the global level, but also at the intermediate level of profitability, solvency and liquidity and managerial aspects. Anyway, these intermediate comparisons are not supplied by basic PROMETHEE methods;

- managerial aspects and profitability aspects are both quite important, therefore both of them receive a relevant weight. However, if a company is profitable, in general, it is also well managed. Consequently, there is the risk of an overevaluation of companies that are both profitable and well managed. In this case, we can say that there is a mutual weakening effect between managerial aspects and profitability aspects. Basic PROMETHEE methods have no possibility to avoid such type of overevaluation. Analogously, other interactions between criteria, such as mutual strengthening and antagonistic effects, cannot be properly handled with basic PROMETHEE methods;

- the financial analysts have not a clear-cut idea about the weights of considered criteria. Again, basic PROMETHEE methods cannot handle in a systematic way these zones of imprecision and hesitation.

Even if there are some extensions of PROMETHEE methods able to deal with the above points singularly (see $[14,15,16,35])$, to the best of our knowledge, there is not any PROMETHEE method taking into consideration the above points all together. Moreover, in so complex problems there is not the possibility to use the very useful visualization support of the GAIA plane ([42]). In fact, the representation on a two dimensional plane supplied by the GAIA plane handles only non-interacting criteria without any consideration of hierarchy of criteria.

In the last years many extensions of PROMETHEE methods have been proposed. They regard weights determination [21], integration with other methods such as Data Envelopment Analysis (DEA) [4, 5], Analytic Network Process (ANP) [37], Analytic Hierarchy Process (AHP) [18] or fuzzy approaches $[11,46]$, integration of quantitative and qualitative information [29], robustness 
of the recommendations as in [19] and, finally, sorting methods [34]. Looking at the appeal of the PROMETHEE methods, witnessed by the many recent papers in the topic mentioned above, we think that it could be interesting an extension of PROMETHEE methods permitting to conjointly handle hierarchy of criteria, interaction between criteria and imprecise definition of weights. Methodologically, the construction of such an extension of the PROMETHEE methods is based on the following tools:

- the Multiple Criteria Hierarchy Process (MCHP) ([12]), which permits to deal with a set of criteria hierarchically structured,

- the bipolar Choquet integral $([22,23])$, which permits to deal with interacting criteria,

- the Robust Ordinal Regression (ROR) $([13,28])$ and the Stochastic Multicriteria Acceptability Analysis (SMAA) ([38]), which permit to deal with imprecision and hesitation in the definition of weights.

- an extension of the GAIA plane $([42])$ which permits, taking into account the plurality of instances of the preference model considered by SMAA (with a procedure different from that one proposed by [30] for the basic PROMETHEE methods), to obtain a graphical visualization of alternatives and criteria, as well as interactions between criteria organized in a hierarchy. In this way, some relevant features (such as clusters, agreements and conflicts between criteria) of the decision problem at hand can be observed.

Of course, MCHP, bipolar Choquet integral, ROR and SMAA, and GAIA plane are not simply put together, but they have to be harmonized within PRQMETHEE methods, taking care that the interrelations properly answer to the requirements of the most complex real world decision problems.

The rest of this paper is organized as follows. Section 2 introduces the fundamental concepts, definitions and notations. Moreover, it reviews the genéral notions of the bipolar Choquet integral, the classical and bipolar PROMETHEE methods and the extension of the bipolar Choquet integral to problems in which the criteria are hierarchically structured. Section 3 introduces the extension of the bipolar PROMETHEE methods and of the GAIA plane technique to the case of interacting criteria organized in a hierarchy. Section 4 concerns considerations regarding the robustness of the recommendations through the ROR and SMAA methodologies as well their visualization on the GAIA plane. Section 5 contains a summary of the main steps involved in the new proposal described by means of a flow chart, while Section 6 describes how to apply the method to a real world problem. Section 7 provides the conclusions and some prospective directions of research. The description of a similar extension for the other PROMETHEE methods is included in the Appendix.

\section{Background}

\subsection{The bipolar Choquet integrals}

The bipolar Choquet integral is based on a bicapacity $[22,23,26]$, being a function $\hat{\mu}: \mathcal{P}(G) \rightarrow$ $[-1,1]$, where $G=\left\{g_{1}, \ldots, g_{m}\right\}^{1}$ is the set of evaluation criteria and $\mathcal{P}(G)=\{(C, D): C, D \subseteq G$ and $C \cap D=\emptyset\}$, such that

- $\hat{\mu}(\emptyset, G)=-1, \quad \hat{\mu}(G, \emptyset)=1, \quad \hat{\mu}(\emptyset, \emptyset)=0$ (boundary conditions),

- for all $(C, D),(E, F) \in \mathcal{P}(G)$, if $C \subseteq E$ and $D \supseteq F$, then $\hat{\mu}(C, D) \leq \hat{\mu}(E, F)$ (monotonicity condition).

\footnotetext{
${ }^{1}$ In the following, for the sake of simplicity, we shall sometimes write criterion $j \in G$ instead of criterion $g_{j} \in G$; that is we shall denote a criterion by its index.
} 
The value $\hat{\mu}(C, D)$, with $(C, D) \in \mathcal{P}(G)$, can be interpreted as the net importance of criteria from $C$ when criteria from $D$ are opposing to them so that, if $\hat{\mu}(C, D)>0(\hat{\mu}(C, D)<0$ or $\hat{\mu}(C, D)=0)$, then criteria in $C$ are more (less or equally) important than those in $D$. Given a vector $x=\left(x_{1}, \ldots, x_{m}\right) \in$ $\mathbb{R}^{m}$ and a bicapacity $\hat{\mu}$ on $\mathcal{P}(G)$, the bipolar Choquet integral of $x$ w.r.t. the bicapacity $\hat{\mu}$ can be defined as

$$
C h^{B}(x, \hat{\mu})=\sum_{j=p}^{m}\left[\left|x_{(j)}\right|-\left|x_{(j-1)}\right|\right] \hat{\mu}\left(\left\{i \in G: x_{i} \geq\left|x_{(j)}\right|\right\},\left\{i \in G: x_{i} \leq-\left|x_{(j)}\right|\right\}\right)
$$

where $(1),(2), \ldots,(m)$ is a permutation of the indices such that $0=\left|x_{(0)}\right| \leq\left|x_{(1)}\right| \leq \ldots \leq\left|x_{(m)}\right|$ and $p=\min _{\substack{j \in\{1, \ldots, m\}: \\\left|x_{(j)}\right|>0}}\{j\}^{2} ;$ moreover, if $x_{(m)}=0 \Rightarrow C h^{B}(x, \hat{\mu})=0$.

$C h^{B}(x, \hat{\mu})$ can be interpreted as the weighted mean of $x \in \mathbb{R}^{m}$ when the importance of criteria is represented by the bicapacity $\hat{\mu}$.

In [25] and [27] the following decomposition of the bicapacity $\hat{\mu}$ has been proposed

$$
\hat{\mu}(C, D)=\mu^{+}(C, D)-\mu^{-}(C, D), \quad \text { for all }(C, D) \in \mathcal{P}(G)
$$

where $\mu^{+}, \mu^{-}: \mathcal{P}(G) \rightarrow[0,1]$ are such that:

$$
\begin{aligned}
& \mu^{+}(G, \emptyset)=1 \text { and } \mu^{+}(\emptyset, B)=0, \quad \forall B \subseteq G \text {, } \\
& \mu^{+}(C, D) \leq \mu^{+}(E, D), \quad \forall C \subseteq E \subseteq G,\left\{\begin{array}{l}
\mu^{-}(C, D) \leq \mu^{-}(C, F),
\end{array}\right. \\
& \mu^{+}(C, D) \geq \mu^{+}(C, F), \quad \forall D \subseteq F \subseteq G, \quad \mu^{-}(C, D) \geq \mu^{-}(E, D), \\
& \left.\begin{array}{l}
\forall B \subseteq G, \\
\forall D \subseteq F \subseteq G, \\
\forall C \subseteq E \subseteq G .
\end{array}\right\}
\end{aligned}
$$

Given a set of alternatives $A=\left\{a_{1}, \ldots, a_{n}\right\}$ and $a, b \in A, \mu^{+}(C, D)$ gives the degree of the importance of criteria in $C$ in favor of the preference of $a$ over $b$ when criteria in $D$ are opposing to it. Conversely, $\mu^{-}(C, D)$ represents the importance of criteria in $D$ opposing to criteria in $C$. As a consequence, as above explained, $\hat{\mu}(C, D)$ is a balance of the-importance of criteria in $C$ (in favor of the preference) and criteria in $D$ (opposing to the same preference).

Using the decomposition of the bicapacity $\hat{\mu}$ defined above, the positive and negative bipolar Choquet integrals of $x \in \mathbb{R}^{m}$ can be defined:

$$
\begin{aligned}
& C h^{B+}(x, \hat{\mu})=\sum_{j=p}^{m}\left[\left|x_{(j)}\right| \hat{-}\left|x_{(j-1)}\right|\right] \mu^{+}\left(\left\{i \in G: x_{i} \geq\left|x_{(j)}\right|\right\},\left\{i \in G: x_{i} \leq-\left|x_{(j)}\right|\right\}\right) \\
& C h^{B-}(x, \hat{\mu})=\sum_{j=p}^{m}\left[\left|x_{(j)} \downarrow-\right| x_{(j-1)} \mid\right] \mu^{-}\left(\left\{i \in G: x_{i} \geq\left|x_{(j)}\right|\right\},\left\{i \in G: x_{i} \leq-\left|x_{(j)}\right|\right\}\right) .
\end{aligned}
$$

where $p=\min _{\substack{j \in\{1, \ldots, m\}: \\ \mid x(\hat{T})>0}}\{j\}$. Supposing that $\bar{x}$ represents the degree of preference of $a$ over $b, C h^{B+}(x, \hat{\mu})$ and $C h^{B-}(x, \hat{\mu})$ give, respectively, how much $a$ is comprehensively preferred to $b$ (considering the reasons in favor of $a$ ) and how much $b$ is preferred to $a$ (considering the reasons against $a$ ). Consequently, the bipolar Choquet integral of $x \in \mathbb{R}^{m}$ is obtained as the difference between the positive and the negative bipolar Choquet integrals.

$$
C h^{B}(x, \hat{\mu})=C h^{B+}(x, \hat{\mu})-C h^{B-}(x, \hat{\mu}) .
$$

In the following we consider 2-additive decomposable bicapacities, such that the next decomposition of $\mu^{+}$and $\mu^{-}$, proposed in [25], holds:

\footnotetext{
${ }^{2}$ For example, if $x=(-1,1,0,2,-2)$, then $\left|x_{(1)}\right|=\left|x_{3}\right|=0 ;\left|x_{(2)}\right|=\left|x_{1}\right|=1 ;\left|x_{(3)}\right|=\left|x_{2}\right|=1 ;\left|x_{(4)}\right|=\left|x_{4}\right|=2$; $\left|x_{(5)}\right|=\left|x_{5}\right|=2$. Therefore, $p=2$.
} 
- $\mu^{+}(C, D)=\sum_{j \in C} a^{+}(\{j\}, \emptyset)+\sum_{\{j, k\} \subseteq C} a^{+}(\{j, k\}, \emptyset)+\sum_{j \in C, k \in D} a^{+}(\{j\},\{k\})$,
- $\mu^{-}(C, D)=\sum_{j \in D} a^{-}(\emptyset,\{j\})+\sum_{\{j, k\} \subseteq D} a^{-}(\emptyset,\{j, k\})+\sum_{j \in C, k \in D} a^{-}(\{j\},\{k\})$.

$a^{+}(\{j\}, \emptyset)$ represents the importance of criterion $j$ when it is in favor of the preference; $a^{+}(\{j, k\}, \emptyset)$ represents the importance of the pair of criteria $\{j, k\}$ when they are in favor of the preference; finally, $a^{+}(\{j\},\{k\})$ represents the antagonistic effect exercised by $k$ over $j$ when $j$ is in favor of the preference and $k$ is against it. The coefficients $a^{-}(\emptyset,\{j\}), a^{-}(\emptyset,\{j, k\})$ and $a^{-}(\{j\},\{k\})$ can be interpreted analogously. $a^{-}(\emptyset,\{j\})$ represents the importance of criterion $j$ when it is against the preference; $a^{-}(\emptyset,\{j, k\})$ represents the importance of the pair of criteria $\{j, k\}$ when they are against the preference; finally, $a^{-}(\{j\},\{k\})$ represents the antagonistic effect exercised by $j$ over $k$ when $k$ is opposing to the preference and $j$ is in favor of it. Let us observe that $a_{j \mid k}^{+} \leq 0$ and $a_{j \mid k}^{-} \leq 0$ for all $j, k=1, \ldots, m, j \neq k$, being representative of the antagonistic effect exereised by $k$ over $j$ in $a_{j \mid k}^{+}$ and of $j$ over $k$ in $a_{j \mid k}^{-}$.

Denoting by $a_{j}^{+}, a_{j k}^{+}$and $a_{j \mid k}^{+}$the coefficients $a^{+}(\{j\}, \emptyset), a^{+}(\{j, k\}, \emptyset)$ and $a^{+}(\{j\},\{k\})$ and by $a_{j}^{-}$, $a_{j k}^{-}$and $a_{j \mid k}^{-}$the coefficients $a^{-}(\emptyset,\{j\}), a^{-}(\emptyset,\{j, k\})$ and $a^{-}(\{j\},\{k\})$, the following conditions have to be satisfied (see [16]):

- monotonicity: $\left\{\begin{array}{l}a_{j}^{+}+\sum_{k \in C} a_{j k}^{+}+\sum_{k \in D} a_{j \mid k}^{+} \geq 0, \forall j \in G, \forall(C \cup\{j\}, D) \in \mathcal{P}(G) \\ a_{j}^{-}+\sum_{k \in D} a_{j k}^{-}+\sum_{k \in C} a_{k \mid j}^{-} \geq 0, \forall j \in G, \forall(C, D \cup\{j\}) \in \mathcal{P}(G)\end{array}\right.$

- normalization: $\left\{\begin{array}{l}\mu^{+}(G, \emptyset)=1 \text {, i.e., } \sum_{j \in G} a_{j}^{+}+\sum_{\{j, k\} \subseteq G} a_{j k}^{+}=1 \\ \mu^{-}(\emptyset, G)=1, \text { i.e., } \sum_{j \in G} a_{j}^{-}+\sum_{\{j, k\} \subseteq G} a_{j k}^{-}=1\end{array}\right.$

Finally, by using the 2-additive decomposition of the bicapacity $\hat{\mu}$ defined above, the positive and negative bipolar Choquet integrals of $x \in \mathbb{R}^{m}$ w.r.t. the bicapacity $\hat{\mu}$ can be redefined as follows $[16]$ :

$$
\begin{gathered}
C h^{B+}(x, \hat{\mu})=\sum_{j \in G, x_{j}>0} a_{j}^{+} x_{j}+\sum_{\substack{j, k \in G, j \neq k \\
x_{j}, x_{k}>0}} a_{j k}^{+} \min \left\{x_{j}, x_{k}\right\}+\sum_{\substack{j, k \in G, j \neq k \\
x_{j}>0, x_{k}<0}} a_{j \mid k}^{+} \min \left\{x_{j},-x_{k}\right\} \\
C h^{B-}(x, \hat{\mu})=-\sum_{j \in x_{j}<0} a_{j}^{-} x_{j}-\sum_{\substack{j, k \in G, j \neq k \\
x_{j}, x_{k}<0}} a_{j k}^{-} \max \left\{x_{j}, x_{k}\right\}-\sum_{\substack{j, k \in G, j \neq k \\
x_{j}>0, x_{k}<0}} a_{j \mid k}^{-} \max \left\{-x_{j}, x_{k}\right\} \\
C^{B}(x, \hat{\mu})=\sum_{j \in x_{j}>0} a_{j}^{+} x_{j}+\sum_{j \in G, x_{j}<0} a_{j}^{-} x_{j}+\sum_{\substack{j, k \in G, j \neq k, x_{j}, x_{k}>0}} a_{j k}^{+} \min \left\{x_{k}, x_{j}\right\}+ \\
\sum_{\substack{j, k \in G, j \neq k, x_{j}, x_{k}<0}} a_{j k}^{-} \max \left\{x_{k}, x_{j}\right\}+\sum_{\substack{j, k \in G, x_{j}>0, x_{k}<0}} a_{j \mid k}^{+} \min \left\{x_{j},-x_{k}\right\}+\sum_{\substack{j, k \in G, x_{j}<0, x_{k}>0}} a_{j \mid k}^{-} \max \left\{x_{j},-x_{k}\right\}
\end{gathered}
$$




\subsection{The classical and bipolar PROMETHEE methods}

PROMETHEE is a family of MCDA methods developed to deal with ranking, choice and sorting problems (see [6] for a survey of the applications of the PROMETHEE methods). In order to apply the PROMETHEE methods (in the following we shall take into account the PROMETHEE I and II only but other PROMETHEE methods are recalled in the Appendix), one has to define for each criterion $g_{j} \in G$ a preference function $p_{j}(a, b)$ expressing, for each couple of alternatives $(a, b) \in A \times A$, the degree of preference of $a$ over $b$ on criterion $g_{j}$. Six different value functions have been proposed in [9]. Here we shall recall the $\mathrm{V}$-shape function $P_{j}(a, b)$ being the most used in practice,

$$
P_{j}(a, b)= \begin{cases}0 & \text { if } \quad d_{j}(a, b) \leq q_{j} \\ \frac{d_{j}(a, b)-q_{j}}{p_{j}-q_{j}} & \text { if } \quad q_{j}<d_{j}(a, b)<p_{j} \\ 1 & \text { if } \quad d_{j}(a, b) \geq p_{j}\end{cases}
$$

where $d_{j}(a, b)=g_{j}(a)-g_{j}(b)$, and $q_{j}, p_{j}$ are the indifference and preference thresholds for criterion $g_{j}$, respectively. Given the positive weights of $m$ criteria $\left(w_{1}, \ldots, w_{m}\right)$ such that $\sum_{j=1}^{m} w_{j}=1$, the comprehensive preference of $a$ over $b$ is given by $\pi(a, b)=\sum_{j=1}^{m} w_{j} P_{j}(a, b)$, while the positive, negative and net flows of $a \in A$ are consequently computed as follows:

$$
\phi^{+}(a)=\frac{1}{n-1} \sum_{b \in A \backslash\{a\}} \pi(a, b), \quad \phi^{-}(a)=\frac{1}{n-1} \sum_{b \in A \backslash\{a\}} \pi(b, a), \quad \phi(a)=\phi^{+}(a)-\phi^{-}(a) .
$$

$\phi^{+}(a)$ represents how much, in average, $a$ is preferred to the other alternatives in $A ; \phi^{-}(a)$ represents how much, in average, the other alternatives in $A$ are preferred to $a$, while $\phi(a)$ represents the comprehensive value of $a$. On one hand, PROMETHEE I provides a partial preorder on $A$ building a preference $P^{I}$, an indifference $I^{I}$ and an incomparability $R^{I}$ relations. In particular, $a P^{I} b$ if $\phi^{+}(a) \geq \phi^{+}(b), \phi^{-}(a) \leq \phi^{-}(b)$ and at least one of the two inequalities is strict; $a I^{I} b$ if $\phi^{+}(a)=\phi^{+}(b)$ and $\phi^{-}(a)=\phi^{-}(b) ; a R^{I} b$ otherwise. On the other hand, PROMETHEE II provides a complete ranking on $A$ by comparing the alternatives w.r.t. their net flow. More in detail, it defines a preference $P^{I I}$ and an indifference $I^{I I}$ relations stating that $a P^{I I} b$ iff $\phi(a)>\phi(b)$, while $a I^{I I} b$ iff $\phi(a)=\phi(b)$.

To extend the PROMETHEE I and PROMETHEE II methods to the bipolar case (for the extension of other classical PROMETHEE methods see the Appendix), for each criterion $g_{j} \in G$, in [16] a bipolar preference function $P_{j}^{B}: A \times A \rightarrow[-1,1]$ has been defined such that:

$$
P_{j}^{B}(a, b)=P_{j}(a, b)-P_{j}(b, a)= \begin{cases}P_{j}(a, b) & \text { if } \quad P_{j}(a, b)>0 \\ -P_{j}(b, a) & \text { if } \quad P_{j}(a, b)=0\end{cases}
$$

Of course a positive value of $P_{j}^{B}(a, b)$ involves the preference of $a$ over $b$ on $j$, while a negative value represents the preference of $b$ over $a$ on the same criterion.

To aggregate the bipolar vector $\left[P_{j}^{B}(a, b)\right]_{j}$, the bipolar Choquet integral presented in the previous section has been applied. In particular, using the 2 -additive decomposition of the bicapacity $\hat{\mu}$, eqs. (5)-(7) can now be rewritten as follows: 


$$
\begin{aligned}
C h^{B+}\left(P^{B}(a, b), \hat{\mu}\right)= & \sum_{j \in G: P_{j}^{B}(a, b)>0} a_{j}^{+} P_{j}^{B}(a, b)+\sum_{\substack{j, k \in G, P_{j}^{B}(a, b)>0, P_{k}^{B}(a, b)>0}} a_{j k}^{+} \min \left\{P_{j}^{B}(a, b), P_{k}^{B}(a, b)\right\}+ \\
+ & \sum_{\substack{j, k \in G, P_{j}^{B}(a, b)>0, P_{k}^{B}(a, b)<0}} a_{j \mid k}^{+} \min \left\{P_{j}^{B}(a, b),-P_{k}^{B}(a, b)\right\}
\end{aligned}
$$

$$
\begin{aligned}
C h^{B-}\left(P^{B}(a, b), \hat{\mu}\right)= & -\sum_{j \in G: P_{j}^{B}(a, b)<0} a_{j}^{-} P_{j}^{B}(a, b)-\sum_{\substack{j, k \in G, P_{j}^{B}(a, b)<0, P_{k}^{B}(a, b)<0}} a_{j k}^{-} \min \left\{P_{j}^{B}(a, b), P_{k}^{B}(a, b)\right\}- \\
- & \sum_{\substack{j, k \in G, P_{j}^{B}(a, b)>0, P_{k}^{B}(a, b)<0}} a_{j \mid k}^{-} \max \left\{-P_{j}^{B}(a, b), P_{k}^{B}(a, b)\right\} .
\end{aligned}
$$

and

$$
C h^{B}\left(P^{B}(a, b), \hat{\mu}\right)=C h^{B+}\left(P^{B}(a, b), \hat{\mu}\right)-C h^{B}+\left(P^{B}(a, b), \hat{\mu}\right) .
$$

$C h^{B+}\left(P^{B}(a, b), \hat{\mu}\right)$ represents the magnitude of the reasons for which $a$ is preferred to $b$, while $C h^{B-}\left(P^{B}(a, b), \hat{\mu}\right)$ represents the magnitude of the reasons against this preference. Therefore, $C h^{B}\left(P^{B}(a, b), \hat{\mu}\right)$ gives an estimate of the overall preference of $a$ over $b$.

In order to impose that the amount of the reasons in favor of the preference of $a$ over $b$ is the same of the amount of the reasons against the preference of $b$ over $a$, technically $C h^{B+}\left(P^{B}(a, b), \hat{\mu}\right)=$ $C h^{B-}\left(P^{B}(b, a), \hat{\mu}\right)$, the following symmetry conditions should be fulfilled (see [16]):

$$
\left\{\begin{array}{l}
a_{j}^{+}=a_{j}^{-}, \text {for each } j \in G, \\
a_{j k}^{+}=a_{j k}^{-}, \text {for each }\{j, k\} \subseteq G, \\
a_{j \mid k}^{+}=a_{k \mid j}^{-}, \text {for each } j, k \in G .
\end{array}\right.
$$

Now, for each alternative $a \in A$, we can define the bipolar positive flow, the bipolar negative flow and the bipolar net flow needed by the bipolar PROMETHEE methods to build the relations of preference, indifference and incomparability

$$
\phi^{B+}(a)=\frac{1}{n-1} \sum_{x \in A \backslash\{a\}} C h^{B+}\left(P^{B}(a, x), \hat{\mu}\right), \quad \phi^{B-}(a)=\frac{1}{n-1} \sum_{x \in A \backslash\{a\}} C h^{B-}\left(P^{B}(a, x), \hat{\mu}\right),
$$

Reminding eq. (12) and using the two equations above, the bipolar net flow $\phi^{B}(a)=\phi^{B+}(a)-\phi^{B-}(a)$ can be written as $\phi^{B}(a)=\frac{1}{n-1} \sum_{x \in A \backslash\{a\}} C h^{B}\left(P^{B}(a, x), \hat{\mu}\right)$.

\subsection{Hierarchical extension of the bipolar positive and negative Choquet integrals}

The MCHP [12] is used when the evaluation criteria are not all located at the same level and they are hierarchically structured. Thus, there is a root criterion (the "comprehensive objective") at level zero, a set of subcriteria of the root criterion at level one, etc. The criteria at the lower level of the hierarchy (which are the "leaves" of the associated tree of criteria) are called elementary. We shall be using the following notation:

- $\mathrm{G}$ is the comprehensive set of criteria (at all levels of the hierarchy), and $g_{\mathbf{0}}$ is the root criterion; - $I_{G}$ is the set of indices of the criteria in $G$; 
- $\mathrm{G}_{\mathrm{E}} \subseteq \mathrm{G}$ is the set of all elementary criteria in $\mathrm{G}$, that are the criteria located at the bottom of the hierarchy;

- $\mathrm{E}_{\mathrm{G}} \subseteq \mathrm{I}_{\mathrm{G}}$ is the set of indices of elementary criteria;

- $g_{\mathbf{r}}$ is a non-elementary criterion if $g_{\mathbf{r}} \in \mathrm{G} \backslash \mathrm{G}_{\mathrm{E}}$;

- $\mathrm{E}\left(g_{\mathbf{r}}\right) \subseteq \mathrm{E}_{\mathrm{G}}$ is the set of indices of all the elementary criteria descending from $g_{\mathbf{r}}$ (it follows that $\left.\mathrm{E}\left(g_{0}\right)=\mathrm{E}_{\mathrm{G}}\right)$;

- given $\mathrm{F} \subseteq \mathrm{G}, \mathrm{E}(\mathrm{F})=\cup_{g_{\mathrm{r}} \in \mathrm{F}} \mathrm{E}\left(g_{\mathbf{r}}\right)$;

- $\mathrm{G}_{\mathbf{r}}^{k} \subseteq \mathrm{G}$ is the set of subcriteria of $g_{\mathbf{r}}$ located at level $k$.

Example 2.1. In order to better explain the proposed extensions, we shall introduce a decision problem that will be discussed in detail in Section 6.

Let us consider the case of financial decision problem of ranking some companies for possible investment. From a risk-return tradeoff point of view, an investment in a company can be measured according to the following main aspects: Profitability (P), Solvency and Liquidity (SEL) and Managerial Performance (MP). Each of these three aspects can be defined by using some financial ratios which can be considered as elementary criteria since they are located at the bottom of the hierarchy (see Figure 1): Gross Profit Ratio (GP/S) and Return on Total Assets (EBIT/S) are subcriteria of Profitability; Debt Ratio (TL/TA) and Current Ratio (CA/CL) are elementary criteria descending from Solvency and Liquidity, while Account Receivable Ratio (AR/S) and Interest Expense Ratio $(I E / S)$ are subcriteria of Managerial Performance. The description of the elementary criteria is provided in Table 1.

Figure 1: Hierarchical structure of criteria considered in the example

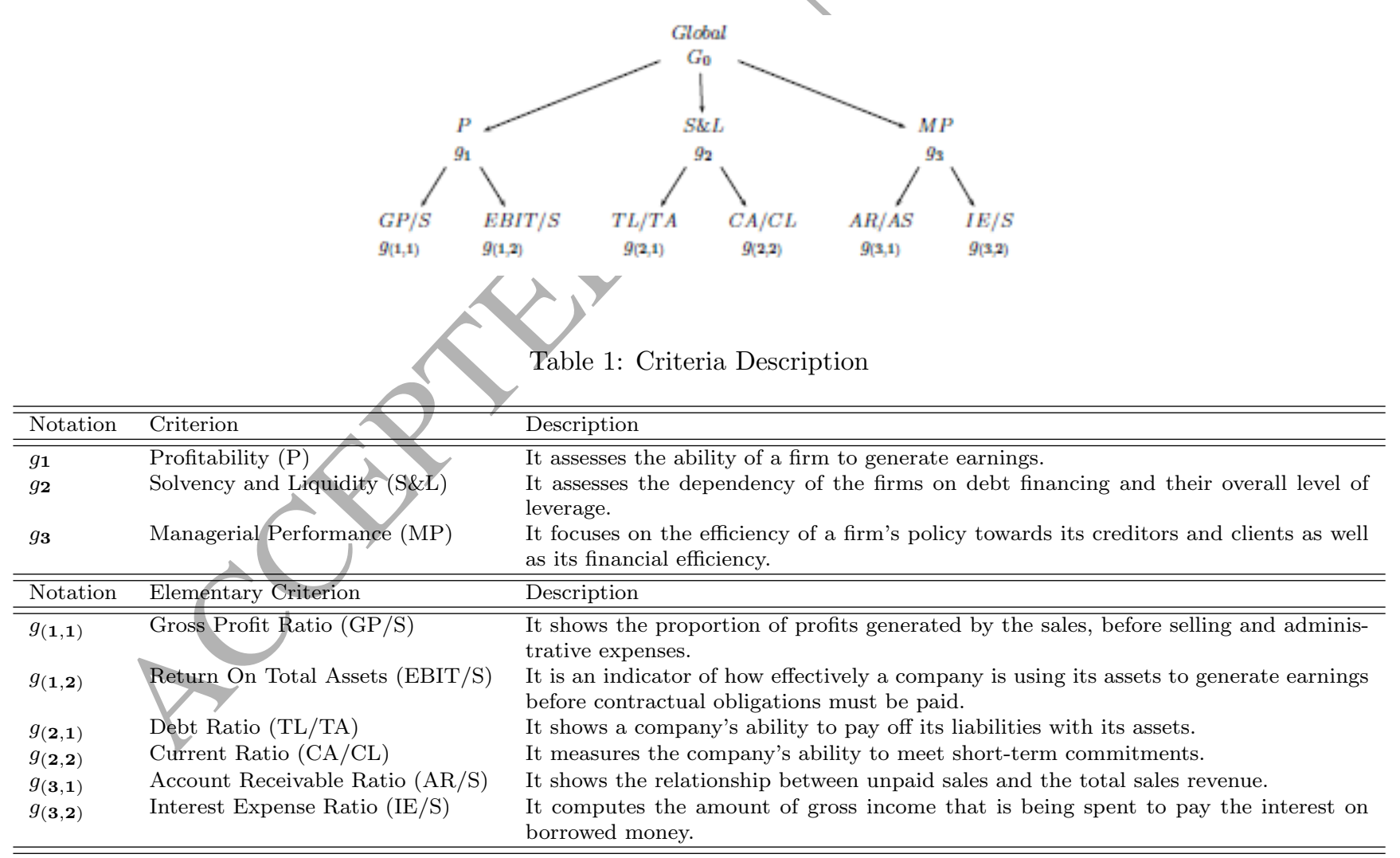

Here, we will extend the bipolar Choquet integral to the hierarchical case to compute, in the next section 3, the bipolar PROMETHEE preference of an alternative $a$ over an alternative $b$ with respect to a particular criterion $g_{\mathbf{r}}$. To do this, we need to introduce a small change in the notation 
used until now. Indeed, while in case of a flat structure of criteria the bicapacity $\hat{\mu}$ was defined on $\mathcal{P}(G)$, where $G$ was the set of criteria $\left\{g_{1}, \ldots, g_{m}\right\}$, from here on, by $\hat{\mu}$ we shall denote a bicapacity defined on $\mathcal{P}\left(\mathrm{G}_{\mathrm{E}}\right)=\left\{(C, D): C, D \subseteq \mathrm{G}_{\mathrm{E}}\right.$ and $\left.C \cap D=\emptyset\right\}$ that is on the set composed of the couples of disjoint subsets of elementary criteria. The same applies to $\mu^{+}$and $\mu^{-}$which will be defined on $\mathcal{P}\left(\mathrm{G}_{\mathrm{E}}\right)$ too.

From a formal point of view, to extend the bipolar Choquet integral of $x \in \mathbb{R}^{\left|\mathrm{G}_{\mathrm{E}}\right|}$ to the hierarchical case, for each non-elementary criterion $g_{\mathbf{r}}$ in the hierarchy, we need to define a bicapacity $\hat{\mu}_{\mathbf{r}}^{k}$ on $\mathcal{P}\left(\mathrm{G}_{\mathbf{r}}^{k}\right)=\left\{(C, D): C, D \subseteq \mathrm{G}_{\mathbf{r}}^{k}\right.$ and $\left.C \cap D=\emptyset\right\}$, such that, for all $(A, B) \in \mathcal{P}\left(\mathrm{G}_{\mathbf{r}}^{k}\right)$,

$$
\hat{\mu}_{\mathbf{r}}^{k}(A, B)=\frac{\hat{\mu}\left(\left\{g_{\mathbf{t}}: \mathbf{t} \in \mathrm{E}(A)\right\},\left\{g_{\mathbf{t}}: \mathbf{t} \in \mathrm{E}(B)\right\}\right)}{\hat{\mu}\left(\left\{g_{\mathbf{t}}: \mathbf{t} \in \mathrm{E}\left(\mathrm{G}_{\mathbf{r}}^{k}\right)\right\}, \emptyset\right)}=\frac{\hat{\mu}\left(\left\{g_{\mathbf{t}}: \mathbf{t} \in \mathrm{E}(A)\right\},\left\{g_{\mathbf{t}}: \mathbf{t} \in \mathrm{E}(B)\right\}\right)}{\hat{\mu}\left(\left\{g_{\mathbf{t}}: \mathbf{t} \in \mathrm{E}\left(g_{\mathbf{r}}\right)\right\}, \emptyset\right)}
$$

where $\hat{\mu}\left(\left\{g_{\mathbf{t}}: \mathbf{t} \in \mathbf{E}\left(g_{\mathbf{r}}\right)\right\}, \emptyset\right) \neq 0$, because, on the contrary, criteria from $\mathrm{G}_{\mathbf{r}}^{k}$ would have null importance and would not be meaningful for the decision problem.

Proposition 2.1. If $\hat{\mu}$ is 2-additive and it satisfies the symmetry conditions in (13), for each nonelementary criterion $g_{\mathbf{r}}$ the following holds:

$$
\hat{\mu}\left(\left\{g_{\mathbf{t}}: \mathbf{t} \in E\left(g_{\mathbf{r}}\right)\right\}, \emptyset\right)=-\hat{\mu}\left(\emptyset,\left\{g_{\mathbf{t}}: \mathbf{t} \in E\left(g_{\mathbf{r}}\right)\right\}\right) .
$$

Proof. By eq. (2),

$$
\hat{\mu}\left(\left\{g_{\mathbf{t}}: \mathbf{t} \in \mathrm{E}\left(g_{\mathbf{r}}\right)\right\}, \emptyset\right)=\mu^{+}\left(\left\{g_{\mathbf{t}}: \mathbf{t} \in \mathrm{E}\left(g_{\mathbf{r}}\right)\right\}, \emptyset\right)-\mu^{-}\left(\left\{g_{\mathbf{t}}: \mathbf{t} \in \mathrm{E}\left(g_{\mathbf{r}}\right)\right\}, \emptyset\right) .
$$

Now, considering a 2-additive decomposition of $\mu^{+}$and $\mu$ and observing that $\mu^{-}\left(\left\{g_{\mathbf{t}}: \mathbf{t} \in \mathbf{E}\left(g_{\mathbf{r}}\right)\right\}, \emptyset\right)=$ 0 , we have that

$$
\mu^{+}\left(\left\{g_{\mathbf{t}}: \mathbf{t} \in \mathrm{E}\left(g_{\mathbf{r}}\right)\right\}, \emptyset\right)-\mu^{-}\left(\left\{g_{\mathbf{t}}: \mathbf{t} \in \mathrm{E}\left(g_{\mathbf{r}}\right)\right\}, \emptyset\right)=\sum_{\mathbf{t} \in \mathbf{E}\left(g_{\mathbf{r}}\right)} a^{+}\left(\left\{g_{\mathbf{t}}\right\}, \emptyset\right)+\sum_{\left\{\mathbf{t}_{1}, \mathbf{t}_{2}\right\} \subseteq \mathrm{E}\left(g_{\mathbf{r}}\right)} a^{+}\left(\left\{g_{\mathbf{t}_{1}}, g_{\mathbf{t}_{2}}\right\}, \emptyset\right)
$$

reminding the symmetry conditions (13), and that $\mu^{+}\left(\emptyset,\left\{g_{\mathbf{t}}: \mathbf{t} \in \mathbf{E}\left(g_{\mathbf{r}}\right)\right\}\right)=0$, we obtain

$$
\begin{gathered}
\sum_{\mathbf{t} \in \mathrm{E}\left(g_{\mathbf{r}}\right)} a^{+}\left(\left\{g_{\mathbf{t}}\right\}, \emptyset\right)+\sum_{\left\{\mathbf{t}_{1}, \mathbf{t}_{2}\right\} \subseteq \mathrm{E}\left(g_{\mathbf{r}}\right)} a^{+}\left(\left\{g_{\mathbf{t}_{1}}, g_{\mathbf{t}_{2}}\right\}, \emptyset\right)=\sum_{\mathbf{t} \in \mathrm{E}\left(g_{\mathbf{r}}\right)} a^{-}\left(\emptyset,\left\{g_{\mathbf{t}}\right\}\right)+\sum_{\left\{\mathbf{t}_{1}, \mathbf{t}_{2}\right\} \subseteq \mathrm{E}\left(g_{\mathbf{r}}\right)} a^{-}\left(\emptyset,\left\{g_{\mathbf{t}_{1}}, g_{\mathbf{t}_{2}}\right\}\right)= \\
\left.=\mu^{-}\left(\emptyset,\left\{g_{\mathbf{t}}: \mathbf{t} \in \mathbf{E}\left(g_{\mathbf{r}}\right)\right\}\right)-\mu^{+}\left(\emptyset,\left\{g_{\mathbf{t}}: \mathbf{t} \in \mathrm{E}\left(g_{\mathbf{r}}\right)\right\}\right)=-\hat{\mu}\left(\emptyset,\left\{g_{\mathbf{t}}: \mathbf{t} \in \mathbf{E}\left(g_{\mathbf{r}}\right)\right\}\right)\right) .
\end{gathered}
$$

Note 2.1. As a consequence of Proposition 2.1, the normalization conditions for the bicapacity $\hat{\mu}_{\mathbf{r}}^{k}$ are satisfied. Indeed:

- $\hat{\mu}_{\mathbf{r}}^{k}(\emptyset, \emptyset)=\frac{\hat{\mu}(\emptyset, \emptyset)}{\hat{\mu}\left(\left\{g_{\mathbf{t}}: \mathbf{t} \in E\left(g_{\mathbf{r}}\right)\right\}, \emptyset\right)}=0$ because $\hat{\mu}(\emptyset, \emptyset)=0$,

- $\hat{\mu}_{\mathbf{r}}^{k}\left(G_{\mathbf{r}}^{k}, \emptyset\right)=\frac{\hat{\mu}\left(\left\{g_{\mathbf{t}}: \mathbf{t} \in E\left(G_{\mathbf{r}}^{k}\right)\right\}, \emptyset\right)}{\hat{\mu}\left(\left\{g_{\mathbf{t}}: \mathbf{t} \in E\left(g_{\mathbf{r}}\right)\right\}, \emptyset\right)}=1$ because $E\left(G_{\mathbf{r}}^{k}\right)=E\left(g_{\mathbf{r}}\right)$,

- $\hat{\mu}_{\mathbf{r}}^{k}\left(\emptyset, G_{\mathbf{r}}^{k}\right)=\frac{\hat{\mu}\left(\emptyset,\left\{g_{\mathbf{t}}: \mathbf{t} \in E\left(G_{\mathbf{r}}^{k}\right)\right\}\right)}{\hat{\mu}\left(\left\{g_{\mathbf{t}}: \mathbf{t} \in E\left(g_{\mathbf{r}}\right)\right\}, \emptyset\right)}=\frac{\hat{\mu}\left(\emptyset,\left\{g_{\mathbf{t}}: \mathbf{t} \in E\left(G_{\mathbf{r}}^{k}\right)\right\}\right)}{-\hat{\mu}\left(\emptyset,\left\{g_{\mathbf{t}}: \mathbf{t} \in E\left(g_{\mathbf{r}}\right)\right\}\right)}=-1$ because $E\left(G_{\mathbf{r}}^{k}\right)=E\left(g_{\mathbf{r}}\right)$ and by Proposition 2.1, 
As the bicapacity $\hat{\mu}$ has been decomposed by means of $\mu^{+}$and $\mu^{-}$, also the bicapacity $\hat{\mu}_{\mathbf{r}}^{k}$ can be decomposed in a similar way,

$$
\hat{\mu}_{\mathbf{r}}^{k}(A, B)=\mu_{\mathbf{r}}^{k,+}(A, B)-\mu_{\mathbf{r}}^{k,-}(A, B)
$$

where $\mu_{\mathbf{r}}^{k,+}, \mu_{\mathbf{r}}^{k,-}: \mathcal{P}\left(\mathrm{G}_{\mathbf{r}}^{k}\right) \rightarrow[0,1]$ and

$$
\begin{array}{ll}
\mu_{\mathbf{r}}^{k,+}(A, B)=\frac{\mu^{+}\left(\left\{g_{\mathbf{t}}: \mathbf{t} \in \mathrm{E}(A)\right\},\left\{g_{\mathbf{t}}: \mathbf{t} \in \mathrm{E}(B)\right\}\right)}{\mu^{+}\left(\left\{g_{\mathbf{t}}: \mathbf{t} \in \mathrm{E}\left(g_{\mathbf{r}}\right)\right\}, \emptyset\right)} & \text { for all }(A, B) \in \mathcal{P}\left(\mathrm{G}_{\mathbf{r}}^{k}\right), \\
\mu_{\mathbf{r}}^{k,-}(A, B)=\frac{\mu^{-}\left(\left\{g_{\mathbf{t}}: \mathbf{t} \in \mathrm{E}(A)\right\},\left\{g_{\mathbf{t}}: \mathbf{t} \in \mathrm{E}(B)\right\}\right)}{\mu^{-}\left(\emptyset,\left\{g_{\mathbf{t}}: \mathbf{t} \in \mathrm{E}\left(g_{\mathbf{r}}\right)\right\}\right)} \quad \text { for all }(A, B) \in \mathcal{P}\left(\mathrm{G}_{\mathbf{r}}^{k}\right) .
\end{array}
$$

with $\mu^{+}\left(\left\{g_{\mathbf{t}}: \mathbf{t} \in \mathbf{E}\left(g_{\mathbf{r}}\right)\right\}, \emptyset\right), \mu^{-}\left(\emptyset,\left\{g_{\mathbf{t}}: \mathbf{t} \in \mathbf{E}\left(g_{\mathbf{r}}\right)\right\}\right) \neq 0$ because, on the contrary, criteria from $\mathrm{E}\left(g_{\mathbf{r}}\right)$ would have null importance and would not be meaningful for the decision problem at hand.

Example 2.2. Let us continue the example introduced above, and let us suppose we wish to compute the bicapacities $\mu_{\mathbf{0}}^{1,+}, \mu_{\mathbf{0}}^{1,-}, \hat{\mu}_{\mathbf{0}}^{1}$ on $G_{0}^{1}=\left\{g_{\mathbf{1}}, g_{\mathbf{2}}, g_{\mathbf{3}}\right\}$. As shown in eqs. (15) and (16), the three bicapacities are dependent on the bicapacities $\mu^{+}$and $\mu^{-}$defined on $\mathcal{P}\left(G_{E}\right)$. For illustrative reasons, let us consider the 2-additive decomposition of $\mu^{+}$and $\mu^{-}$shown in Table 2; we considered the coefficients $a^{+}$only because the analogous coefficients $a^{-}$can be obtained by $a^{+}$due to the symmetry conditions in 13. Moreover, all coefficients a not specified in Table 2 are fixed equal to zero.

\begin{tabular}{|c|c|c|c|c|c|}
\hline & $a^{+}(\{\cdot\},\{\cdot\})$ & & $a^{a^{+}(\{\cdot\},\{\cdot\})}$ & & $a^{+}(\{\cdot\},\{\cdot\})$ \\
\hline$\left(\left\{g_{(\mathbf{1}, \mathbf{1})}\right\}, \emptyset\right)$ & 0.281 & $\left(\left\{g_{(\mathbf{1}, \mathbf{1})}, g_{(\mathbf{3}, \mathbf{1})}\right\}, \emptyset\right)$ & 0043 & $\left(\left\{g_{(\mathbf{2}, \mathbf{2})}, g_{(\mathbf{3}, \mathbf{1})}\right\}, \emptyset\right)$ & -0.028 \\
\hline$\left(\left\{g_{(\mathbf{1}, \mathbf{2})}\right\}, \emptyset\right)$ & 0.282 & $\left(\left\{g_{(\mathbf{1}, \mathbf{1})}, g_{(\mathbf{3}, \mathbf{2})}\right\}, \emptyset\right)$ & 005 & $\left(\left\{g_{(\mathbf{2}, \mathbf{2})}, g_{(\mathbf{3}, \mathbf{2})}\right\}, \emptyset\right)$ & -0.077 \\
\hline$\left(\left\{g_{(\mathbf{2}, \mathbf{1})}\right\}, \emptyset\right)$ & 0.218 & & -0.076 & $\left(\left\{g_{(\mathbf{1}, \mathbf{1})}\right\},\left\{g_{(\mathbf{2}, \mathbf{1})}\right\}\right)$ & -0.015 \\
\hline$\left(\left\{g_{(\mathbf{2}, \mathbf{2})}\right\}, \emptyset\right)$ & 0.173 & & 0.083 & $\left(\left\{g_{(\mathbf{1}, \mathbf{1})}\right\},\left\{g_{(\mathbf{2}, \mathbf{2})}\right\}\right)$ & -0.0700 \\
\hline$\left(\left\{g_{(\mathbf{3}, \mathbf{1})}\right\}, \emptyset\right)$ & 0.121 & $\left(\left\{g_{(\mathbf{2}, \mathbf{1})}, g_{(\mathbf{2}, \mathbf{2})}\right\}, \emptyset\right)$ & -0.053 & $\left(\left\{g_{(\mathbf{1}, \mathbf{1})}\right\},\left\{g_{(\mathbf{3}, \mathbf{1})}\right\}\right)$ & -0.036 \\
\hline$\left(\left\{g_{(\mathbf{3}, \mathbf{2})}\right\}, \emptyset\right)$ & 0.234 & & -0.015 & $\left(\left\{g_{(\mathbf{1}, \mathbf{2})}\right\},\left\{g_{(\mathbf{2}, \mathbf{1})}\right\}\right)$ & -0.049 \\
\hline$\left(\left\{g_{(\mathbf{1}, \mathbf{1})}, g_{(\mathbf{1}, \mathbf{2})}\right\}, \emptyset\right)$ & -0.032 & $\left(\left\{g_{(2,1)}, g_{(3,2)}\right\}, \emptyset\right)$ & -0.102 & $\left(\left\{g_{(\mathbf{1}, \mathbf{2})}\right\},\left\{g_{(\mathbf{2}, \mathbf{2})}\right\}\right)$ & -0.051 \\
\hline
\end{tabular}

In the following, we shall show how to compute $\mu_{\mathbf{0}}^{1,+}\left(\left\{g_{\mathbf{1}}\right\},\left\{g_{\mathbf{2}}\right\}\right)$ and $\mu_{\mathbf{0}}^{1,-}\left(\left\{g_{\mathbf{1}}\right\},\left\{g_{\mathbf{2}}\right\}\right)$. From eqs.

Table 2: Example of the 2-additive decomposition of $\mu^{+}$

(15) and (16), we have

$$
\mu_{\mathbf{0}}^{1,+}\left(\left\{g_{\mathbf{1}}\right\},\left\{g_{\mathbf{2}}\right\}\right)=\frac{\mu^{+}\left(\left\{g_{\mathbf{t}}: \mathbf{t} \in E\left(g_{\mathbf{1}}\right)\right\},\left\{g_{\mathbf{t}}: \mathbf{t} \in E\left(g_{\mathbf{2}}\right)\right\}\right)}{\mu^{+}\left(\left\{g_{\mathbf{t}}: \mathbf{t} \in E\left(g_{\mathbf{0}}\right)\right\}, \emptyset\right)}
$$

and

$$
\mu_{0}^{1 .}-\left(\left\{g_{\mathbf{1}}\right\},\left\{g_{\mathbf{2}}\right\}\right)=\frac{\mu^{-}\left(\left\{g_{\mathbf{t}}: \mathbf{t} \in E\left(g_{\mathbf{1}}\right)\right\},\left\{g_{\mathbf{t}}: \mathbf{t} \in E\left(g_{\mathbf{2}}\right)\right\}\right)}{\mu^{-}\left(\emptyset,\left\{g_{\mathbf{t}}: \mathbf{t} \in E\left(g_{\mathbf{0}}\right)\right\}\right)} .
$$

In consequence of the 2-additive decomposition of $\mu^{+}$and $\mu^{-}$provided in Section 2.1, we have that:

- $\mu^{+}\left(\left\{g_{\mathbf{t}}: \mathbf{t} \in E\left(g_{\mathbf{1}}\right)\right\},\left\{g_{\mathbf{t}}: \mathbf{t} \in E\left(g_{\mathbf{2}}\right)\right\}\right)=$

$=\sum_{\mathbf{t} \in E\left(g_{1}\right)} a^{+}\left(\left\{g_{\mathbf{t}}\right\}, \emptyset\right)+\sum_{\mathbf{t}_{\mathbf{1}}, \mathbf{t}_{\mathbf{2}} \in E\left(g_{1}\right)} a^{+}\left(\left\{g_{\mathbf{t}_{\mathbf{1}}}, g_{\mathbf{t}_{2}}\right\}, \emptyset\right)+\sum_{\substack{\mathbf{t}_{\mathbf{1}} \in E\left(g_{1}\right) \\ \mathbf{t}_{\mathbf{2}} \in E\left(g_{\mathbf{2}}\right)}} a^{+}\left(\left\{g_{\mathbf{t}_{1}}\right\},\left\{g_{\mathbf{t}_{2}}\right\}\right)=a^{+}\left(\left\{g_{(\mathbf{1}, \mathbf{1})}\right\}, \emptyset\right)+$

$+a^{+}\left(\left\{g_{(\mathbf{1}, \mathbf{2})}\right\}, \emptyset\right)+a^{+}\left(\left\{g_{(\mathbf{1}, \mathbf{1})}, g_{(\mathbf{1}, \mathbf{2})}\right\}, \emptyset\right)+a^{+}\left(\left\{g_{(\mathbf{1}, \mathbf{1})}\right\},\left\{g_{(\mathbf{2}, \mathbf{1})}\right\}\right)+a^{+}\left(\left\{g_{(\mathbf{1}, \mathbf{1})}\right\},\left\{g_{(\mathbf{2}, \mathbf{2})}\right\}\right)+$

$+a^{+}\left(\left\{g_{(\mathbf{1}, \mathbf{2})}\right\},\left\{g_{(\mathbf{2}, \mathbf{1})}\right\}\right)+a^{+}\left(\left\{g_{(\mathbf{1}, \mathbf{2})}\right\},\left\{g_{(\mathbf{2}, \mathbf{2})}\right\}\right)=0.281+0.282-0.032-0.015-0.070-0.049-$

$-0.051=0.344$. 
- $\mu^{+}\left(\left\{g_{\mathbf{t}}: \mathbf{t} \in E\left(g_{\mathbf{0}}\right)\right\}, \emptyset\right)=\mu^{+}\left(\left\{g_{(\mathbf{1}, \mathbf{1})}, g_{(\mathbf{1}, \mathbf{2})}, g_{(\mathbf{2}, \mathbf{1})}, g_{(\mathbf{2}, \mathbf{2})}, g_{(\mathbf{3}, \mathbf{1})}, g_{(\mathbf{3}, \mathbf{2})}\right\}, \emptyset\right)=1$ in consequence of the normalization constraints on $\mu^{+}$,

- $\mu^{-}\left(\left\{g_{\mathbf{t}}: \mathbf{t} \in E\left(g_{1}\right)\right\},\left\{g_{\mathbf{t}}: \mathbf{t} \in E\left(g_{\mathbf{2}}\right)\right\}\right)=$

$=\sum_{\mathbf{t} \in E\left(g_{\mathbf{2}}\right)} a^{-}\left(\emptyset,\left\{g_{\mathbf{t}}\right\}\right)+\sum_{\mathbf{t}_{\mathbf{1}}, \mathbf{t}_{\mathbf{2}} \in E\left(g_{\mathbf{2}}\right)} a^{-}\left(\emptyset,\left\{g_{\mathbf{t}_{1}}, g_{\mathbf{t}_{2}}\right\}\right)+\sum_{\substack{\mathbf{t}_{1} \in E\left(g_{1}\right) \\ \mathbf{t}_{2} \in E\left(g_{\mathbf{2}}\right)}} a^{-}\left(\left\{g_{\mathbf{t}_{1}}\right\},\left\{g_{\mathbf{t}_{2}}\right\}\right)=a^{-}\left(\emptyset,\left\{g_{(\mathbf{2}, \mathbf{1})}\right\}\right)+$

$+a^{-}\left(\emptyset,\left\{g_{(\mathbf{2}, \mathbf{2})}\right\}\right)+a^{-}\left(\emptyset,\left\{g_{(\mathbf{2}, \mathbf{1})}, g_{(\mathbf{2}, \mathbf{2})}\right\}\right)+a^{-}\left(\left\{g_{(\mathbf{1}, \mathbf{1})}\right\},\left\{g_{(\mathbf{2}, \mathbf{1})}\right\}\right)+a^{-}\left(\left\{g_{(\mathbf{1}, \mathbf{1})}\right\},\left\{g_{(\mathbf{2}, \mathbf{2})}\right\}\right)+$

$+a^{-}\left(\left\{g_{(\mathbf{1}, \mathbf{2})}\right\},\left\{g_{(\mathbf{2}, \mathbf{1})}\right\}\right)+a^{-}\left(\left\{g_{(\mathbf{1}, \mathbf{2})}\right\},\left\{g_{(\mathbf{2}, \mathbf{2})}\right\}\right)={ }^{3} a^{+}\left(\left\{g_{(\mathbf{2}, \mathbf{1})}\right\}, \emptyset\right)+a^{+}\left(\left\{g_{(\mathbf{2}, \mathbf{2})}\right\}, \emptyset\right)+$

$+a^{+}\left(\left\{g_{(\mathbf{2}, \mathbf{1})}, g_{(\mathbf{2}, \mathbf{2})}\right\}, \emptyset\right)+a^{+}\left(\left\{g_{(\mathbf{2}, \mathbf{1})}\right\},\left\{g_{(\mathbf{1}, \mathbf{1})}\right\}\right)+a^{+}\left(\left\{g_{(\mathbf{2}, \mathbf{2})}\right\},\left\{g_{(\mathbf{1}, \mathbf{1})}\right\}\right)+a^{+}\left(\left\{g_{(\mathbf{2}, \mathbf{1})}\right\},\left\{g_{(\mathbf{1}, \mathbf{2})}\right\}\right)+$

$+a^{+}\left(\left\{g_{(\mathbf{2}, \mathbf{2})}\right\},\left\{g_{(\mathbf{1 , 2})}\right\}\right)=0.218+0.173-0.053=0.338$.

- $\mu^{-}\left(\emptyset,\left\{g_{\mathbf{t}}: \mathbf{t} \in E\left(g_{\mathbf{0}}\right)\right\}\right)=\mu^{-}\left(\emptyset,\left\{g_{(\mathbf{1}, \mathbf{1})}, g_{(\mathbf{1}, \mathbf{2})}, g_{(\mathbf{2}, \mathbf{1})}, g_{(\mathbf{2}, \mathbf{2})}, g_{(\mathbf{3}, \mathbf{1})}, g_{(\mathbf{3}, \mathbf{2})}\right\}\right)=1$ in consequence of the normalization constraints on $\mu^{-}$.

Analogously, one can compute the values of the bicapacity for each pair of subsets of criteria $(A, B) \in$ $\mathcal{P}\left(G_{0}^{1}\right)$ as shown in Table 3.

Table 3: Values of the bicapacities $\mu_{\mathbf{0}}^{1,+}, \mu_{\mathbf{0}}^{1,-}$ and $\hat{\mu}_{\mathbf{0}}^{1}$ considering the 2-additive decomposition of $\mu^{+}$given in Table 2

\begin{tabular}{|c|c|c|c|c|c|c|c|c|c|c|c|}
\hline$(\cdot, \cdot)$ & $\mu_{0}^{1,+}(\cdot, \cdot)$ & $\overline{\mu_{0}^{1,-}(\cdot, \cdot)}$ & $\hat{\mu}_{0}^{1}(\cdot, \cdot)$ & $(\cdot, \cdot)$ & $\mu_{0}^{1,+}(\cdot, \cdot)$ & $\mu_{0}^{1,-}(\cdot \cdot)$ & $\hat{\mu}_{0}^{1}(\cdot, \cdot)$ & $(\cdot, \cdot)$ & $\mu_{0}^{1,+}(\cdot, \cdot)$ & $\mu_{0}^{1,-}(\cdot, \cdot)$ & $\hat{\mu}_{\mathbf{0}}^{1}(\cdot, \cdot)$ \\
\hline$\left(g_{1}, \emptyset\right)$ & 0.531 & 0 & 0.531 & $\left(\left\{g_{2}\right\},\left\{g_{1}\right\}\right)$ & 0.338 & 0.344 & -0.005 & $\left(\left\{g_{1}, g_{3}\right\},\left\{g_{2}\right\}\right)$ & 0.698 & 0.338 & 0.359 \\
\hline$\left(g_{\mathbf{2}}, \emptyset\right)$ & 0.338 & 0 & 0.338 & $\left(\left\{g_{2}\right\},\left\{g_{3}\right\}\right)$ & 0.338 & 0.356 & 0.018 & $\left(\left\{g_{2}, g_{3}\right\},\left\{g_{1}\right\}\right)$ & 0.471 & 0.307 & 0.163 \\
\hline$\left(g_{\mathbf{3}}, \emptyset\right)$ & 0.356 & 0 & 0.356 & $\left(\left\{g_{3}\right\},\left\{g_{1}\right\}\right)$ & & & -0.138 & $\left(\emptyset,\left\{g_{1}\right\}\right)$ & 0 & 0.531 & -0.531 \\
\hline$\left(\left\{g_{1}, g_{2}\right\}, \emptyset\right)$ & 0.870 & 0 & 0.870 & $\left(\left\{g_{3}\right\},\left\{g_{2}\right\}\right)$ & & 0.338 & 0.017 & $\left(\emptyset,\left\{g_{2}\right\}\right)$ & 0 & 0.338 & -0.338 \\
\hline$\left(\left\{g_{1}, g_{3}\right\}, \emptyset\right)$ & 0.885 & 0 & 0.885 & $\left(\left\{g_{1}\right\}\right.$ & 0.307 & 0.471 & -0.163 & $\left(\emptyset,\left\{g_{3}\right\}\right)$ & 0 & 0.356 & -0.356 \\
\hline$\left(\left\{g_{2}, g_{3}\right\}, \emptyset\right)$ & 0.471 & 0 & 0.471 & & 0.338 & 0.698 & -0.359 & $\left(\emptyset,\left\{g_{1}, g_{2}\right\}\right)$ & 0 & 0.870 & -0.870 \\
\hline$\left(\left\{g_{1}\right\},\left\{g_{2}\right\}\right)$ & 0.344 & 0.338 & 0.006 & & 0.356 & 0.833 & -0.477 & $\left(\emptyset,\left\{g_{1}, g_{\mathbf{3}}\right\}\right)$ & 0 & 0.885 & -0.885 \\
\hline$\left(\left\{g_{1}\right\},\left\{g_{\mathbf{3}}\right\}\right)$ & 0.494 & 0.356 & 0.138 & & 0.833 & 0.356 & 0.477 & $\left(\emptyset,\left\{g_{2}, g_{\mathbf{3}}\right\}\right)$ & 0 & 0.471 & -0.471 \\
\hline$(\emptyset, \emptyset)$ & 0 & 0 & & \}$, \emptyset)$ & 1 & 0 & 1 & $\left(\emptyset,\left\{g_{1}, g_{2}, g_{3}\right\}\right)$ & 0 & 1 & -1 \\
\hline
\end{tabular}

Considering eqs. (15) and (16), the positive and negative bipolar Choquet integral of $x \in \mathbb{R}^{\left|\mathrm{E}_{G}\right|}$ on a particular node $g_{\mathbf{r}}$ can be computed as follows:

$$
\begin{aligned}
C h_{\mathbf{r}}^{B+}\left(x, \hat{\mu}_{\mathbf{r}}^{k}\right) & =\frac{C h^{B+}\left(x_{\mathbf{r}}, \hat{\mu}\right)}{\mu^{+}\left(\left\{g_{\mathbf{t}}: \mathbf{t} \in \mathrm{E}\left(g_{\mathbf{r}}\right)\right\}, \emptyset\right)} \\
C h_{\mathbf{r}}^{B-}\left(x, \hat{\mu}_{\mathbf{r}}^{k}\right) & =\frac{C h^{B-}\left(x_{\mathbf{r}}, \hat{\mu}\right)}{\mu^{-}\left(\emptyset,\left\{g_{\mathbf{t}}: \mathbf{t} \in \mathbf{E}\left(g_{\mathbf{r}}\right)\right\}\right)}
\end{aligned}
$$

where $x_{\mathbf{r}}$ is a fictitious vector having the same components of $x$ on the indices corresponding to the elementary criteria descending from $g_{\mathbf{r}}$ and zero on the others.

\footnotetext{
${ }^{3}$ Remember that $a^{+}\left(\left\{g_{\mathbf{t}}\right\}, \emptyset\right)=a^{-}\left(\left\{g_{\mathbf{t}}\right\}, \emptyset\right)$ for all $g_{\mathbf{t}} \in \mathrm{G}_{\mathrm{E}} ; a^{+}\left(\left\{g_{\mathbf{t}_{1}}, g_{\mathbf{t}_{2}}\right\}, \emptyset\right)=a^{-}\left(\emptyset,\left\{g_{\mathbf{t}_{1}}, g_{\mathbf{t}_{2}}\right\}\right)$ and $a^{+}\left(\left\{g_{\mathbf{t}_{1}}\right\},\left\{g_{\mathbf{t}_{2}}\right\}\right)=a^{-}\left(\left\{g_{\mathbf{t}_{2}}\right\},\left\{g_{\mathbf{t}_{1}}\right\}\right)$ for all $g_{\mathbf{t}_{1}}, g_{\mathbf{t}_{2}} \in \mathrm{G}_{\mathrm{E}}$
} 
Proposition 2.2. If $\hat{\mu}$ is 2-additive and it satisfies the symmetry conditions in 13, for each nonelementary criterion $g_{\mathbf{r}}$, the following holds:

$$
\mu^{+}\left(\left\{g_{\mathbf{t}}: \mathbf{t} \in E\left(g_{\mathbf{r}}\right)\right\}, \emptyset\right)=\mu^{-}\left(\emptyset,\left\{g_{\mathbf{t}}: \mathbf{t} \in E\left(g_{\mathbf{r}}\right)\right\}\right)
$$

Proof. Considering a 2-additive bicapacity and in consequence of the symmetry conditions in eq. (13), we have that:

$$
\begin{aligned}
& \mu^{+}\left(\left\{g_{\mathbf{t}}: \mathbf{t} \in \mathrm{E}\left(g_{\mathbf{r}}\right)\right\}, \emptyset\right)=\sum_{\mathbf{t} \in \mathrm{E}\left(g_{\mathbf{r}}\right)} a^{+}\left(\left\{g_{\mathbf{t}}\right\}, \emptyset\right)+\sum_{\left\{\mathbf{t}_{1}, \mathbf{t}_{2}\right\} \subseteq \mathrm{E}\left(g_{\mathbf{r}}\right)} a^{+}\left(\left\{g_{\mathbf{t}_{1}}, g_{\mathbf{t}_{2}}\right\}, \emptyset\right)= \\
& =\sum_{\mathbf{t} \in \mathrm{E}\left(g_{\mathbf{r}}\right)} a^{-}\left(\emptyset,\left\{g_{\mathbf{t}}\right\}\right)+\sum_{\left\{\mathbf{t}_{1}, \mathbf{t}_{2}\right\} \subseteq \mathrm{E}\left(g_{\mathbf{r}}\right)} a^{-}\left(\emptyset,\left\{g_{\mathbf{t}_{1}}, g_{\mathbf{t}_{2}}\right\}\right)=\mu^{-}\left(\emptyset,\left\{g_{\mathbf{t}}: \mathbf{t} \in \mathrm{E}\left(g_{\mathbf{r}}\right)\right\}\right) .
\end{aligned}
$$

The bipolar Choquet integral of $x$ on a node $g_{\mathbf{r}}$ can be defined as

$$
C h_{\mathbf{r}}^{B}\left(x, \hat{\mu}_{\mathbf{r}}^{k}\right)=C h_{\mathbf{r}}^{B+}\left(x, \hat{\mu}_{\mathbf{r}}^{k}\right)-C h_{\mathbf{r}}^{B-}\left(x, \hat{\mu}_{\mathbf{r}}^{k}\right)
$$

that, on the basis of the positive and negative bipolar Choquet integral of $x$ w.r.t. $g_{\mathbf{r}}$ given in eqs. (17) and (18) and considering Proposition 2.2, can be written as

$$
C h_{\mathbf{r}}^{B}\left(x, \hat{\mu}_{\mathbf{r}}^{k}\right)=\frac{C h^{B}\left(x_{\mathbf{r}}, \hat{\mu}\right)}{\hat{\mu}\left(\left\{g_{\mathbf{t}}: \mathbf{t} \in \mathbb{E}\left(g_{\mathbf{r}}\right)\right\}, \emptyset\right)} .
$$

\section{Bipolar PROMETHEE methods extended to the MCHP}

While in the previous section we proposed an extension of the bipolar Choquet integral to the case of a set of criteria structured in a hierarchical way, in this section, we shall extend the bipolar PROMETHEE method to the case of interacting criteria organized in a hierarchy.

On the basis of the comprehensive positive and negative preference of $a$ over $b$ given in eqs. (10) and (11) and considering the definitions of the bipolar positive, negative and comprehensive Choquet integral w.r.t. a criterion $g_{\mathbf{r}}$ in the hierarchy, we can give the following definition.

Definition 3.1. Given $a, b \in A$ and a non-elementary criterion $g_{\mathbf{r}}$ in the hierarchy, we can define:

- The comprehensive positive preference of a over $b$ on $g_{\mathbf{r}}$ :

$$
C h_{\mathbf{r}}^{B+}\left(P^{B}(a, b), \hat{\mu}_{\mathbf{r}}^{k}\right)=\frac{C h^{B+}\left(P_{\mathbf{r}}^{B}(a, b), \hat{\mu}\right)}{\mu^{+}\left(\left\{g_{\mathbf{t}}: \mathbf{t} \in E\left(g_{\mathbf{r}}\right)\right\}, \emptyset\right)},
$$

- The comprehensive negative preference of a over $b$ on $g_{\mathbf{r}}$ :

$$
C h_{\mathbf{r}}^{B-}\left(P^{B}(a, b), \hat{\mu}_{\mathbf{r}}^{k}\right)=\frac{C h^{B-}\left(P_{\mathbf{r}}^{B}(a, b), \hat{\mu}\right)}{\mu^{-}\left(\emptyset,\left\{g_{\mathbf{t}}: \mathbf{t} \in E\left(g_{\mathbf{r}}\right)\right\}\right)}
$$

- The comprehensive preference of a over $b$ on $g_{\mathbf{r}}$ :

$$
C h_{\mathbf{r}}^{B}\left(P^{B}(a, b), \hat{\mu}_{\mathbf{r}}^{k}\right)=\frac{C h^{B}\left(P_{\mathbf{r}}^{B}(a, b), \hat{\mu}\right)}{\hat{\mu}\left(\left\{g_{\mathbf{t}}: \mathbf{t} \in E\left(g_{\mathbf{r}}\right)\right\}, \emptyset\right)},
$$


In all three definitions, $P_{\mathbf{r}}^{B}(a, b)$ is the vector belonging to $\mathbb{R}^{\left|E\left(g_{\mathbf{r}}\right)\right|}$ such that

$$
\left(P_{\mathbf{r}}^{B}(a, b)\right)_{\mathbf{t}}= \begin{cases}P_{\mathbf{t}}^{B}(a, b) & \text { if } \mathbf{t} \in E\left(g_{\mathbf{r}}\right), \\ 0 & \text { if } \mathbf{t} \notin E\left(g_{\mathbf{r}}\right) .\end{cases}
$$

Example 3.1. Let's go back to the example introduced in Section 2.3. The evaluations of the eight most capitalized European pharmaceutical companies on the six elementary criteria are shown in Table 4, while the indifference and preference thresholds for all elementary criteria are given in Table 5 .

Table 4: Evaluations of the eight pharmaceutical companies on the considered elementary criteria

\begin{tabular}{l|cccccc}
\hline \hline Pharma Companies & GP/S & EBIT/S & TL/TA & CA/CL & AR/S & IE/S \\
\hline \hline Glaxo & 0.664 & 0.209 & 0.904 & 1.236 & 0.160 & 0.027 \\
Sanofi & 0.687 & 0.054 & 0.430 & 1.482 & 0.212 & 0.007 \\
Novartis & 0.660 & 0.067 & 0.414 & 0.964 & 0.162 & 0.013 \\
Roche Holding & 0.708 & 0.177 & 0.723 & 1.186 & 0.170 & 0.018 \\
AstraZeneca & 0.818 & 0.068 & 0.692 & 1.077 & 0.188 & 0.016 \\
Bayer & 0.543 & 0.085 & 0.672 & 1.403 & 0.214 & 0.016 \\
Merck & 0.683 & 0.048 & 0.664 & 0.783 & 0.213 & 0.024 \\
Novo Nordisk & 0.850 & 0.539 & 0.488 & 1.349 & 0.143 & 0.001 \\
\hline \hline Preference direction & Incr. & Incr. & Decr. & Incr. & Decr. & Decr. \\
\hline \hline \multicolumn{7}{l}{} \\
& & & & & & \\
\end{tabular}

Table 5: Indifference and preference thresholds for the elementary criteria

\begin{tabular}{cccc|cccc|cccc}
\hline \hline$q_{(\mathbf{1}, \mathbf{1})}$ & $p_{(\mathbf{1}, \mathbf{1})}$ & $q_{(\mathbf{1}, \mathbf{2})}$ & $p_{(\mathbf{1}, \mathbf{2})}$ & $q_{(\mathbf{2}, \mathbf{1})}$ & $p_{(\mathbf{2}, \mathbf{1})}$ & $q_{(\mathbf{2}, \mathbf{2})}$ & $p_{(\mathbf{2}, \mathbf{2})}$ & $q_{(\mathbf{3}, \mathbf{1})}$ & $p_{(\mathbf{3}, \mathbf{1})}$ & $q_{(\mathbf{3}, \mathbf{2})}$ & $p_{(\mathbf{3}, \mathbf{2})}$ \\
0.02 & 0.06 & 0.01 & 0.03 & 0.05 & 0.15 & 0.10 & 0.20 & 0.01 & 0.02 & 0.003 & 0.006 \\
\hline \hline
\end{tabular}

Considering Sanofi and Novartis we can compute the bipolar vector $P^{B}$ (Sanofi, Novartis) at the global level as well as on all three macrocriteria:

- $P^{B}($ Sanofi, Novartis $)=(0.1711,-0.1379,0,1,-1,1)$,

- $P_{1}^{B}($ Sanofi, Novartis $)=(0.1711,-0.1379,0,0,0,0)$,

- $P_{2}^{B}($ Sanofi, Novartis $)=(0,0,0,1,0,0)$,

- $P_{\mathbf{3}}^{B}$ (Sanofi, Novartis $)=(0,0,0,0,-1,1)$.

Now, let us consider the 2-additive decomposition of a bicapacity $\hat{\mu}$ given in Table 2. To compute the comprehensive positive preference of Sanofi over Novartis on Profitability, by eq. (21), we have that

$$
\begin{gathered}
\qquad C h_{\mathbf{1}}^{B+}\left(P^{B}(\text { Sanofi, Novartis }), \hat{\mu}_{\mathbf{1}}^{2}\right)=\frac{C h^{B+}\left(P_{\mathbf{1}}^{B}(\text { Sanofi, Novartis }), \hat{\mu}\right)}{\mu^{+}\left(\left\{g_{\mathbf{t}}: \mathbf{t} \in E\left(g_{\mathbf{1}}\right)\right\}, \emptyset\right)}= \\
=\frac{C h^{B+}((0.1711,-0.1379,0,0,0,0), \hat{\mu})}{\mu^{+}\left(\left\{g_{(\mathbf{1}, \mathbf{1})}, g_{(\mathbf{1}, \mathbf{2})}\right\}, \emptyset\right)} .
\end{gathered}
$$

By eq. (10) and considering the 2-additive decomposition of $\mu^{+}$we get

- $C h^{B+}((0.1711,-0.1379,0,0,0,0), \hat{\mu})=$

$$
=0.1711 \cdot a^{+}\left(\left\{g_{(\mathbf{1}, \mathbf{1})}\right\}, \emptyset\right)+\min \{0.1711,0.1379\} \cdot a^{+}\left(\left\{g_{(\mathbf{1}, \mathbf{1})}\right\},\left\{g_{(\mathbf{1}, \mathbf{2})}\right\}\right)=0.1711 \cdot 0.281=0.048 \text {. }
$$


- $\mu^{+}\left(\left\{g_{(\mathbf{1}, \mathbf{1})}, g_{(\mathbf{1}, \mathbf{2})}\right\}, \emptyset\right)=$

$=a^{+}\left(\left\{g_{(\mathbf{1}, \mathbf{1})}\right\}, \emptyset\right)+a^{+}\left(\left\{g_{(\mathbf{1}, \mathbf{2})}\right\}, \emptyset\right)+a^{+}\left(\left\{g_{(\mathbf{1}, \mathbf{1})}, g_{(\mathbf{1}, \mathbf{2})}\right\}, \emptyset\right)=0.281+0.282-0.032=0.531$.

Consequently, $\mathrm{Ch}_{\mathbf{1}}^{B+}\left(P^{B}(\right.$ Sanofi, Novartis $\left.), \hat{\mu}_{1}^{2}\right)=\frac{0.048}{0.531}=0.090$.

By using the definitions above, the comprehensive negative preference and the comprehensive preference of Sanofi over Novartis at a global level as well as at partial level can be computed (see Table $6)$.

Table 6: Comprehensive positive and negative preference of Sanofi over Novartis at global level as well as at partial level

\begin{tabular}{c|ccc}
\hline \hline & $C h_{\mathbf{r}}^{B+}\left(P^{B}(\right.$ Sanofi, Novartis $\left.), \hat{\mu}_{\mathbf{r}}^{2}\right)$ & $C h_{\mathbf{r}}^{B-}\left(P^{B}(\right.$ Sanofi, Novartis $\left.), \hat{\mu}_{\mathbf{r}}^{2}\right)$ & $C h_{\mathbf{r}}^{B}\left(P^{B}(\right.$ Sanofi, Novartis $\left.), \hat{\mu}_{\mathbf{r}}^{2}\right)$ \\
\hline \hline $\mathbf{r}=(\mathbf{1})$ & 0.0906 & 0.0734 & 0.0172 \\
$\mathbf{r}=(\mathbf{2})$ & 0.5117 & 0 & 0.5117 \\
$\mathbf{r}=(\mathbf{3})$ & 0.6581 & 0.3419 & 0.3163 \\
\hline \hline
\end{tabular}

On the basis of the previous definitions, the positive, negative and net bipolar flows w.r.t. a criterion $g_{\mathbf{r}}$ can be computed.

Definition 3.2. Given $a \in A$ and a non-elementary criterion $g_{\mathbf{r}}$, we can define:

- The bipolar positive flow of a on $g_{\mathbf{r}}$ :

$$
\phi_{\mathbf{r}}^{B+}(a)=\frac{1}{n-1} \sum_{x \in A \backslash\{a\}} C h_{\mathbf{r}}^{B+}\left(P^{B}(a, x), \hat{\mu}_{\mathbf{r}}^{k}\right)=\frac{1}{n-1} \sum_{x \in A \backslash\{a\}} \frac{C h^{B+}\left(P_{\mathbf{r}}^{B}(a, x), \hat{\mu}\right)}{\mu^{+}\left(\left\{g_{\mathbf{t}}: \mathbf{t} \in E\left(g_{\mathbf{r}}\right)\right\}, \emptyset\right)}
$$

- The bipolar negative flow of a on $g_{\mathbf{r}}$ :

$$
\phi_{\mathbf{r}}^{B-}(a)=\frac{1}{n-1} \sum_{x \in A \backslash\{a\}}{C h_{\mathbf{r}}^{B-}}^{B}\left(P^{B}(a, x), \hat{\mu}_{\mathbf{r}}^{k}\right)=\frac{1}{n-1} \sum_{x \in A \backslash\{a\}} \frac{C h^{B-}\left(P_{\mathbf{r}}^{B}(a, x), \hat{\mu}\right)}{\mu^{-}\left(\emptyset,\left\{g_{\mathbf{t}}: \mathbf{t} \in E\left(g_{\mathbf{r}}\right)\right\}\right)}
$$

- The bipolar net flow of a on $g_{\mathbf{r}}$ :

$$
\begin{aligned}
\phi_{\mathbf{r}}^{B}(a) & =\phi_{\mathbf{r}}^{B+}(a)-\phi_{\mathbf{r}}^{B-}(a)=\frac{1}{n-1} \sum_{x \in A \backslash\{a\}}\left[C h_{\mathbf{r}}^{B+}\left(P^{B}(a, x), \hat{\mu}_{\mathbf{r}}^{k}\right)-C h_{\mathbf{r}}^{B-}\left(P^{B}(a, x), \hat{\mu}_{\mathbf{r}}^{k}\right)\right]= \\
& =\frac{1}{n-1} \sum_{x \in A \backslash\{a\}} C h_{\mathbf{r}}^{B}\left(P^{B}(a, x), \hat{\mu}_{\mathbf{r}}^{k}\right)=\frac{1}{n-1} \sum_{x \in A \backslash\{a\}} \frac{C h^{B}\left(P_{\mathbf{r}}^{B}(a, x), \hat{\mu}\right)}{\hat{\mu}\left(\left\{g_{\mathbf{t}}: \mathbf{t} \in E\left(g_{\mathbf{r}}\right)\right\}, \emptyset\right)}
\end{aligned}
$$

Using definitions 3.1 and 3.2, the extension of the bipolar PROMETHEE I and II methods to the case of criteria organized in a hierarchical way (that we shall call hierarchical bipolar PROMETHEE I and II methods) can be provided. In particular, given $a, b \in A$ and a non-elementary criterion $g_{\mathbf{r}}$,

- the hierarchical bipolar PROMETHEE I method defines a preference $\left(\mathcal{P}_{\mathbf{r}, B}^{I}\right)$, an indifference $\left(\mathcal{I}_{\mathbf{r}, B}^{I}\right)$ and an incomparability $\left(\mathcal{R}_{\mathbf{r}, B}^{I}\right)$ relation as follows:

$-a \mathcal{P}_{\mathbf{r}, B}^{I} b$ iff $\phi_{\mathbf{r}}^{B+}(a) \geq \phi_{\mathbf{r}}^{B+}(b), \phi_{\mathbf{r}}^{B-}(a) \leq \phi_{\mathbf{r}}^{B-}(b)$ and at least one of the two inequalities is strict, 
- $b \mathcal{P}_{\mathbf{r}, B}^{I} a$ iff $\phi_{\mathbf{r}}^{B+}(b) \geq \phi_{\mathbf{r}}^{B+}(a), \phi_{\mathbf{r}}^{B-}(b) \leq \phi_{\mathbf{r}}^{B-}(a)$ and at least one of the two inequalities is strict,

$-a \mathcal{I}_{\mathbf{r}, B}^{I} b$ iff $\phi_{\mathbf{r}}^{B+}(a)=\phi_{\mathbf{r}}^{B+}(b)$ and $\phi_{\mathbf{r}}^{B-}(a)=\phi_{\mathbf{r}}^{B-}(b)$,

- $a \mathcal{R}_{\mathbf{r}, B}^{I} b$, otherwise.

- the hierarchical bipolar PROMETHEE II method defines a preference $\left(\mathcal{P}_{\mathbf{r}, B}^{I I}\right)$ and an indifference $\left(\mathcal{I}_{\mathbf{r}, B}^{I I}\right)$, only:

$-a \mathcal{P}_{\mathbf{r}, B}^{I I} b$ iff $\phi_{\mathbf{r}}^{B}(a)>\phi_{\mathbf{r}}^{B}(b)$,

$-a \mathcal{I}_{\mathbf{r}, B}^{I I} b$ iff $\phi_{\mathbf{r}}^{B}(a)=\phi_{\mathbf{r}}^{B}(b)$.

Example 3.2. Continuing the previous example, we shall show how to compute the relations defined above on profitability for the hierarchical bipolar PROMETHEE I and II methods. Let us consider the 2-additive decomposition of the bicapacity $\hat{\mu}$ given in Table 2 and the evaluations of the eight companies on the eight elementary subcriteria shown in Table 4. The bipolar positive, negative and net flow of each alternative on criterion $g_{1}$ shown in Table 7 can be computed. One can observe that Roche

Table 7: Bipolar positive, negative and net flows on Profitability

\begin{tabular}{l|ccc}
\hline \hline & $\phi_{\mathbf{1}}^{B+}(\cdot)$ & $\phi_{\mathbf{1}}^{B-}(\cdot)$ & $\phi_{\mathbf{1}}^{B(}()$ \\
\hline \hline Glaxo & 0.5230 & 0.2693 & 0.2537 \\
Sanofi & 0.0942 & 0.4791 & -0.3849 \\
Novartis & 0.1194 & 0.4629 & -0.3435 \\
Roche Holding & 0.5486 & 0.2946 & 0.2540 \\
AstraZeneca & 0.5007 & 0.2708 & 0.2299 \\
Bayer & 0.2032 & 0.7313 & -0.5218 \\
Merck & 0.0808 & 0.5217 & -0.4409 \\
Novo Nordisk & 0.9256 & 0 & 0.9256 \\
\hline \hline
\end{tabular}

is preferred to Glaxo on profitability considering the hierarchical bipolar PROMETHEE II method (Roche $\mathcal{P}_{\mathbf{1}, B}^{I I} G$ laxo), while the two companies are incomparable considering the bipolar PROMETHEE I method (Roche $\mathcal{R}_{1, B}^{I}$ Glaxo). The preferences between all pairs of companies obtained by the new hiearchical bipolar PROMETHEE methods are shown in figure 2.

\subsection{GAIA plane for the bipolar PROMETHEE methods}

Since PROMETHEE methods are often implemented along with the GAIA plane [42], we propose an extension of this visualization tool to the case of bipolar PROMETHEE methods with criteria organized in a hierarchy taking also into account the plurality of instances for the preference model considered by SMAA.

As known, the purpose of this technique is to obtain a graphical representation of the matrix of the partial criteria net flows by using the Principal Component Analysis (PCA) [33, 2, 50] to reduce the dimensionality. In particular, in the classical case, the GAIA plane permits to visualize in a two dimensional space the projections of criteria and alternatives which look as vectors and points, respectively. In many applications of the GAIA plane, the PCA is based on the covariance matrix. In this case, if there are large differences between the variances of the single criteria net flows then, those single criteria net flow which have the largest variance tend to dominate the first principal 


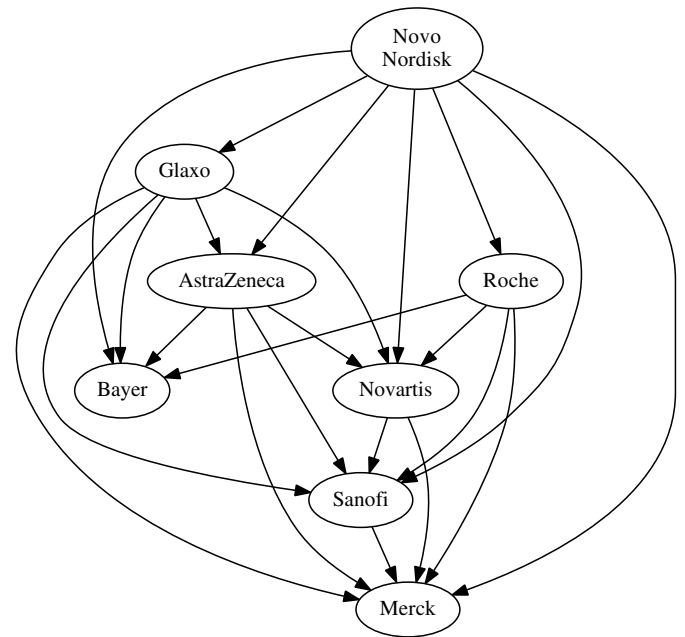

(a) Hierarchical bipolar PROMETHEE I method

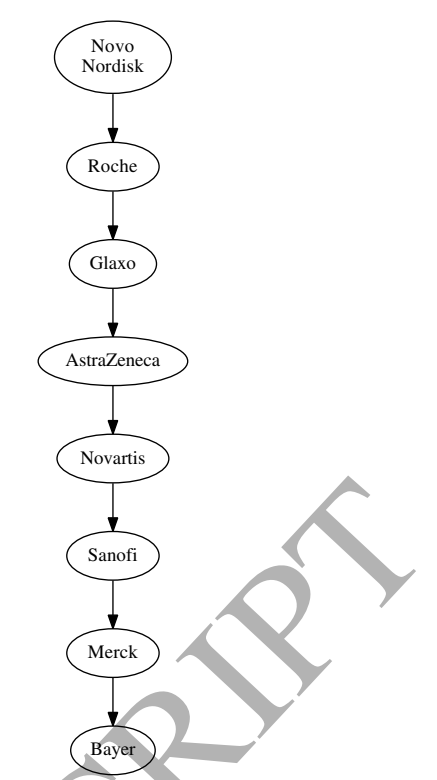

(b) Hier-

archical

bipolar

PROMETHEE

II method

Figure 2: Preference relations obtained by the hierarchical bipolar PROMETHEE methods on Profitability

component. For this reason, here we apply the PCA to the correlation matrix ${ }^{4}$ in order to avoid an unreasonably large impact on the principal components by few variables treating all variables on an equal footing.

To apply this visualization technique to the bipolar case, we start by decomposing the net flow w.r.t. the bipolar PROMETHEE method defined in Section 2.2 in terms of parameters useful for the visual tool, as follows:

$$
\phi^{B}(a)=\frac{1}{n-1} \sum_{x \in A \backslash\{a\}}\left[\sum_{j \in G} a_{j}^{+} P_{j}^{B}(a, x)+\sum_{(j, k) \in S y n} a_{j k}^{+} \alpha_{j k}(a, x)+\sum_{(j, k) \in \text { Ant }} a_{j \mid k}^{+} \beta_{j \mid k}(a, x)\right]
$$

where:

- Syn $\subseteq\{(j, k): j, k \in G, j<k\}=\left\{\left(j_{1}^{s}, k_{1}^{s}\right), \ldots,\left(j_{q}^{s}, k_{q}^{s}\right)\right\}$ is the set of non-ordered pairs of criteria for which the DM declared that there is interaction and therefore $a_{j k}^{+} \neq 0$,

- Ant $\subseteq\{(j, k): j, k \in G, j \neq k\}=\left\{\left(j_{1}^{a}, k_{1}^{a}\right), \ldots,\left(j_{t}^{a}, k_{t}^{a}\right)\right\}$ is the set of ordered pairs of criteria for which the DM declared that there is antagonism and therefore $a_{j \mid k}^{+}<0$,

$$
\alpha_{j k}(a, x)= \begin{cases}\min \left\{P_{j}^{B}(a, x), P_{k}^{B}(a, x)\right\} & \text { if } P_{j}^{B}(a, x)>0, P_{k}^{B}(a, x)>0 \\ \max \left\{P_{j}^{B}(a, x), P_{k}^{B}(a, x)\right\} & \text { if } P_{j}^{B}(a, x)<0, P_{k}^{B}(a, x)<0 \\ 0 & \text { otherwise }\end{cases}
$$

\footnotetext{
${ }^{4}$ In this case the vectors of loadings (principal component coefficients) consist of the eigenvectors of the correlation matrix.
} 


$$
\beta_{j \mid k}(a, x)= \begin{cases}\min \left\{P_{j}^{B}(a, x),-P_{k}^{B}(a, x)\right\} & \text { if } P_{j}^{B}(a, x)>0, P_{k}^{B}(a, x)<0 \\ \max \left\{-P_{j}^{B}(a, x), P_{k}^{B}(a, x)\right\} & \text { if } P_{k}^{B}(a, x)>0, P_{j}^{B}(a, x)<0 \\ 0 & \text { otherwise. }\end{cases}
$$

Through this decomposition, we are considering interactions and antagonistic effects as criteria for which the evaluations of alternatives are given by the functions defined in eqs. (25) and (26), respectively.

Now, instead of the classical matrix containing the single criterion net flows, we have to consider a matrix $\boldsymbol{\Phi}$ which, as in the classic case, takes into account the preferences given by the generalized criteria but with the additional information given by the interactions between criteria as well as the antagonistic effects between them. The size of the matrix $\Phi$ depends on the information provided by the DM in the sense that it contains the columns related to interactions and antagonistic effects only if the DM provides them. This means that the matrix $\boldsymbol{\Phi}$ does not contain the columns for which the related information is not provided ${ }^{5}$. We consider the matrix $\boldsymbol{\Phi}$ as input data to perform the PCA. The general form of the matrix $\boldsymbol{\Phi}$ is shown in Table 8, where

$$
\begin{aligned}
\phi_{j}^{B}(a) & =\frac{1}{n-1} \sum_{x \in A} P_{j}^{B}(a, x) \\
\phi_{j k}^{B}(a) & =\frac{1}{n-1} \sum_{x \in A} \alpha_{j k}(a, x) \\
\phi_{j \mid k}^{B}(a) & =\frac{1}{n-1} \sum_{x \in A} \beta_{j \mid k}(a, x)
\end{aligned}
$$

Table 8: Matrix of the parameters involved in the extension of the GAIA method. Partial bipolar preference index for each criterion and parameters representing interaction and antagonistic effects between criteria.

\begin{tabular}{l|lllllllll} 
& $\phi_{1}^{B}(\cdot)$ & $\ldots$ & $\phi_{m}^{B}(\cdot)$ & $\phi_{j_{1}^{s} k_{1}^{s}}^{B}(\cdot)$ & $\ldots$ & $\phi_{j_{q}^{s} k_{q}^{s}}^{B}(\cdot)$ & $\phi_{j_{1}^{a} \mid k_{1}^{a}}^{B}(\cdot)$ & $\ldots$ & $\phi_{j_{t}^{a} \mid k_{t}^{a}}^{B}(\cdot)$ \\
\hline$a_{1}$ & $\phi_{1}^{B}\left(a_{1}\right)$ & $\ldots$ & $\phi_{m}^{B}\left(a_{1}\right)$ & $\phi_{j_{1}^{s} k_{1}^{s}}\left(a_{1}\right)$ & $\ldots$ & $\phi_{j_{q}^{s} k_{q}^{s}}\left(a_{1}\right)$ & $\phi_{j_{1}^{a} \mid k_{1}^{a}}^{B}\left(a_{1}\right)$ & $\ldots$ & $\phi_{j_{t}^{a} \mid k_{t}^{a}}\left(a_{1}\right)$ \\
$\vdots$ & $\vdots$ & $\vdots$ & $\vdots$ & $\gamma_{1}$ & $\vdots$ & $\vdots$ & $\vdots$ & $\vdots$ & $\vdots$ \\
$a_{n}$ & $\phi_{1}^{B}\left(a_{n}\right)$ & $\ldots$ & $\phi_{m}^{B}\left(a_{n}\right)$ & $\phi_{j_{1}^{s} k_{1}^{s}}^{B}\left(a_{n}\right)$ & $\ldots$ & $\phi_{j_{q}^{s} k_{q}^{s}}^{B}\left(a_{n}\right)$ & $\phi_{j_{1}^{a} \mid k_{1}^{a}}^{B}\left(a_{n}\right)$ & $\ldots$ & $\phi_{j_{t}^{a} \mid k_{t}^{a}}^{B}\left(a_{n}\right)$
\end{tabular}

Note that the vector $\phi^{B}=\left[\phi^{B}\left(a_{1}\right), \ldots, \phi^{B}\left(a_{n}\right)\right]$ containing the net flow of alternatives from $A$ can be written in terms of the product between the matrix $\boldsymbol{\Phi}$ and the vector â containing the decomposition of the bicapacity $\hat{\mu}$ as shown in eq. (30).

$$
\hat{\mathbf{a}}=\left(a_{1}^{+}, \ldots, a_{m}^{+}, a_{j_{1}^{s} k_{1}^{s}}^{+}, \ldots, a_{j_{q}^{s} k_{q}^{s}}^{+}, a_{j_{1}^{a} \mid k_{1}^{a}}^{+}, \ldots, a_{j_{t}^{a} \mid k_{t}^{a}}^{+}\right) \text {. }
$$

We apply the principal component analysis solving the following problem:

$$
\begin{aligned}
& \max \mathbf{u}^{\prime} C \mathbf{u} \text { subject to } \\
& \mathbf{u}^{\prime} \mathbf{u}=1 .
\end{aligned}
$$

where $\mathbf{u}$ is a unit vector, while

$$
C=\frac{\Phi^{S t d^{\prime}} \Phi^{S t d}}{m+q+t}
$$

${ }^{5}$ The maximum size of the matrix is $n \times\left[m+\left(\begin{array}{c}m \\ 2\end{array}\right)+D_{m, 2}\right]$, where $m$ is the number of considered criteria and $n$ is the number of alternatives. Note that each element of $\boldsymbol{\Phi}$ belongs to $[-1,1]$. 
is the variance-covariance matrix of centered and standardized data

$$
\Phi^{S t d}=\left[\phi_{r}^{s t d}\left(a_{i}\right), r=1, \ldots, m, j_{1}^{s} k_{1}^{s}, \ldots, j_{q}^{s} k_{q}^{s}, j_{1}^{a} k_{1}^{a}, \ldots, j_{t}^{a} k_{t}^{a}\right]
$$

which generic element is obtained as

$$
\phi_{r}^{S t d}\left(a_{i}\right)=\frac{\phi_{r}^{B}\left(a_{i}\right)-M_{r}}{\sigma_{r}}
$$

with $M_{r}$ and $\sigma_{r}$ denoting the the mean and the standard deviation of the elements in the $r$-th column of matrix $\Phi$, respectively, that is

$$
M_{r}=\frac{\sum_{i=1}^{n} \phi_{r}^{B}\left(a_{i}\right)}{n}, \quad \sigma_{r}=\sqrt{\frac{\sum_{i=1}^{n}\left(\phi_{r}^{B}\left(a_{i}\right)-M_{r}\right)^{2}}{n}} .
$$

Using the Lagrange theorem this amounts to solve the system

$$
\left\{\begin{array}{l}
C \mathbf{u}=\lambda \mathbf{u} \\
\mathbf{u}^{\prime} \mathbf{u}=1
\end{array}\right.
$$

After this, the two largest eigenvalues $\lambda_{1}$ and $\lambda_{2}$ of $C$ and the corresponding eigenvectors $\mathbf{u}_{1}$ and $\mathbf{u}_{2}$ have to be calculated. Then each point

$$
\mathbf{x}=\left(x_{1}^{+}, \ldots, x_{m}^{+}, x_{j_{1}^{s} k_{1}^{s}}^{+}, \ldots, x_{j_{q}^{s} k_{q}^{s}}^{+}, x_{j_{1}^{a} \mid k_{1}^{a}}^{+}, \ldots, x_{j_{t}^{a} \mid k_{t}^{a}}^{+}\right) .
$$

is projected on the GAIA plane to the point having coordinates $\left(\mathbf{u}_{1}^{\prime} \mathbf{x}, \mathbf{u}_{2}^{\prime} \mathbf{x}\right)$. The explained variance of the GAIA plane, that is the percentage of information kept by the projection, is given by

$$
\delta=\frac{\lambda_{1}+\lambda_{2}}{\sum_{r=1}^{m+q+t} \lambda_{r}}
$$

where $\lambda_{r}, r=1, \ldots, m+q+t$, is the $r$-th eigenvalue of $C$. The value of $\delta$ is in general larger than $60 \%$ and most times larger than $85 \%$ 18].

In this framework, each alternative $a \in A$ is represented by the projection on the GAIA plane of the following vector

$$
\phi^{\mathbf{B}}(\mathbf{a})=\left[\phi_{1}^{B}(a), \ldots, \phi_{m}^{B}(a), \phi_{j_{1}^{s} k_{1}^{s}}^{B}(a), \ldots, \phi_{j_{q}^{s} k_{q}^{s}}^{B}(a), \phi_{j_{1}^{a} \mid k_{1}^{a}}^{B}(a), \ldots, \phi_{j_{t}^{a} k_{t}^{a}}^{B}(a)\right] .
$$

Analogously, importance of criteria, positive and negative interactions and antagonism between pairs of criteria are projected on the GAIA plane as follows:

- criterion $g_{j} \in G$ is represented by the axis linking the origin of the plane with the projection of the vector $\mathbf{x}$ which components are all null with the exception of $x_{j}$ being equal to 1 ,

- the negative or positive interaction between criteria $\left(j_{e}^{s}, k_{e}^{s}\right), e=1, \ldots, q$, is represented by the axis linking the origin of the plane with the projection of the vector $\mathbf{x}$ which components are all null with the exception of $x_{j_{e}^{s}, k_{e}^{s}}$ being equal to 1 ,

- the antagonism between criteria $\left(j_{f}^{a}, k_{f}^{a}\right), f=1, \ldots, t$ is represented by the axis linking the origin of the plane with the projection of the vector $\mathbf{x}$ which components are all null with the exception of $x_{j_{f}^{a}, k_{f}^{a}}$ being equal to 1 . 
We briefly remark some properties of the GAIA plane:

- the length of the projection of a criterion, interaction or the antagonism between a pair of criteria, is representative of its capacity to discriminate between the alternatives;

- projections of criteria, positive or negative interactions or antagonisms between pairs of criteria oriented in similar directions express similar preference. Analogously, projections of criteria, positive or negative interactions or antagonisms having opposite directions represent opposite preferences;

- criteria, synergies or antagonisms between pairs of criteria which projections appear orthogonal are uncorrelated in terms of preferences;

- the closer the points representing two alternatives on the GAIA plane the more similar the alternatives are;

- an alternative $a \in A$ has a good evaluation with respect to criterion $g_{j} \in G$ if it is located in the same direction of $g_{j}$ and, instead, it has a bad evaluation if it is located in the opposite direction of $g_{j}$. More precisely, in case of location in the same (opposite) direction of $g_{j}$, the longer the projection of the point representing an alternative $a \in A$ on the axis of a criterion $g_{j} \in G$, the better (worse) its evaluation on $g_{j}$; similar interpretations hold with respect to interaction and antagonism of pairs of of criteria.

With respect to the overall evaluation of an alternatives from $A$, the projection of vector â on the GAIA plane constitutes the PROMETHEE decision axis corresponding to the projection of the weight vector for the classical PROMETHEE methods. In general, the longer the projection of the point representing an alternative $a \in A$ on the décision axis, the greater the net flow $\phi^{B}(a)$.

The above general principles of the GAIA plane suffer of some exceptions due to the approximation of the projection of all the variables related to single criteria, interactions and antagonistic effects on the 2-dimensional plane. Anyway, the greater the variance $\delta$ explained by the first two components of PCA, the greater the information preserved and, consequently, the less these exceptions are.

In case of a hierarchy of criteria, one can get a GAIA plane for each non elementary criterion $g_{\mathbf{r}} \in \mathrm{G} \backslash \mathrm{G}_{\mathbf{E}}$ by narrowing the above analysis to the elementary criteria $g_{\mathbf{t}}, \mathbf{t} \in \mathbf{E}\left(g_{\mathbf{r}}\right)$, descending from $g_{\mathbf{r}}$.

Example 3.3. Let us continue the example introduced above. Let us consider the 2-additive decomposable bicapacity whose decomposition vector $\hat{\mathbf{a}}$ is presented in Table 9. In this case, the PCA is applied to 21 variables (6 criteria, 10 interactions and 5 antagonistic effects). Figure $3(a),(b)$ and (c) show axis representing single criteria $g_{j} \in G$, pairs of criteria $(j, k) \in$ Syn for which there is a positive or negative interaction, and pairs of criteria $(j, k) \in$ Ant for which there is an antagonistic effect, respectively. For reasons of space, in Figure 3, numbers 1,2,3,4,5 and 6 represent elementary criteria $G P / S, E B I T / S, T L / T A, C A / C L, A R / S$ and $I E / S$, respectively. The points representing the alternatives as well as the PROMETHEE decision axis, indicated with the label "Capacity", are shown in all the three Figure $3(a),(b)$ and (c). One can observe the following points:

- Figure 3(a), having an explained variance $\delta=68.72 \%$ (as well as Figure 3(b) and (c) that show different elements, but on the same GAIA plane), shows that all criteria are quite discriminating because their axis are quite long. Moreover, criteria $C A / C L, T L / T A, I E / S$, on one hand, and $G P / S, E B I T / S$ and $A R / S$, on the other hand, express similar preferences. Novo Nordisk is in the same direction of all criteria and has long projections on their axis, so that it has good evaluations on all criteria. On the contrary, Merck is in the opposite direction of all criteria and has relatively long projections on their axis, so that it has bad evaluations on all criteria. 
AstraZeneca and Roche Holding are relatively close on the GAIA plane and this shows that they have similar evaluations on all the criteria.

- Figure 3(b) displays a certain proximity of the axis representing interaction between the pairs of criteria (TL/TA, CA/CL), (CA/CL, IE/S) and (TL/TA, IE/S), showing that they express similar aspects in the decision problem. Analogously, there is a certain similarity between the interaction of the pairs $(C A / C L, A R / S)$, (TL/TA, AR/S), (GP/GS,IE/S), (EBIT/S, IE/S), on one hand, and the pairs $(G P / S, E B I T / S),(G P / S, A R / S),(E B I T / S, A R / S)$, on the other hand.

- Figure 3(c) shows a certain similarity of the antagonism for the pairs $(G P / S \mid T L / T A)$ and $(G P / S \mid C A / C L)$, on one hand, and the pairs $(E B I T / S \mid T L / T A)$ and $(E B I T / S \mid C A / C L)$. Instead the antagonism of the pair $(G P / S \mid A R / S)$ has its own specific features as shown by the location of its axis which is quite isolated with respect to the other axis representing pairs of antagonistic criteria.

- Comparing the points representing alternatives with the PROMETHEE decision axis "Capacity" one can see that Novo Nordisk is by far the best alternative, while Bayer and Meck are the worst ones. Indeed, all these alternatives have a long projection on the "Capacity" axis. However, Novo Nordisk is in the same direction of "Capacity" axis, while Bayer and Merck are in the opposite direction.

- Figure 3(d), having an explained variance $\delta=99.47 \%$, shows that with respect to the nonelementary criterion Solvency and Liquidity, the two elementary criteria CA/CL and TL/TA are both discriminant (because of their long axis) and independent (because of the orthogonality of their axis), while the interaction between the two criteria is less discriminant (because of the relatively short axis). Alternatives are quite different between them (because of their quite scattered location), with the exception of a relative similarity between Novo Nordisk and Sanofi, on one hand, and Roche Holding and AstraZeneca, on the other hand. With respect to the overall evaluation related to Solvency and Liquidity, one can see that the best alternatives are Novo Nordisk and Sanofi while the worst alternatives are Merck and Glaxo. All these four alternatives have long projections on the PROMETHEE decision axis "Capacity", but Novo Nordisk and Sanofi are in the same direction of the "Capacity" axis, while Merck and Glaxo are in the opposite direction.

Table 9: 2-additive decomposable bicapacity considered in the example.

\begin{tabular}{|c|c|c|c|c|c|c|c|c|c|c|}
\hline$a_{1}^{+}$ & $a_{2}^{+}$ & $a_{3}^{+}$ & $a_{4}^{+}$ & $a_{5}^{+}$ & $a_{6}^{+}$ & $a_{12}^{+}$ & $a_{15}^{+}$ & $a_{16}^{+}$ & $a_{25}^{+}$ & $a_{26}^{+}$ \\
\hline 0.259 & 0.296 & 0.185 & 0.222 & 0.148 & 0.222 & -0.037 & 0.037 & -0.074 & -0.037 & 0.037 \\
\hline$a_{34}^{+}$ & $a_{35}^{+}$ & $a_{36}^{+}$ & $a_{45}^{+}$ & $a_{46}^{+}$ & $a_{1 \mid 3}^{+}$ & $a_{1 \mid 4}^{+}$ & $a_{1 \mid 5}^{+}$ & $a_{2 \mid 3}^{+}$ & $a_{2 \mid 4}^{+}$ & \\
\hline-0.074 & -0.037 & -0.037 & -0.037 & -0.074 & -0.037 & -0.037 & -0.037 & -0.055 & -0.055 & \\
\hline
\end{tabular}

\section{Exploring the results through SMAA and ROR}

\subsection{Providing an indirect preference information}

The proposed hierarchical bipolar PROMETHEE methods can be easily considered as a generalization of the classical PROMETHEE methods. Indeed, it is able to take into account several aspect that are not considered in the classical PROMETHEE methods such as the hierarchical structure of criteria, the interactions between criteria as well as the antagonistic effects between them. Anyway, 


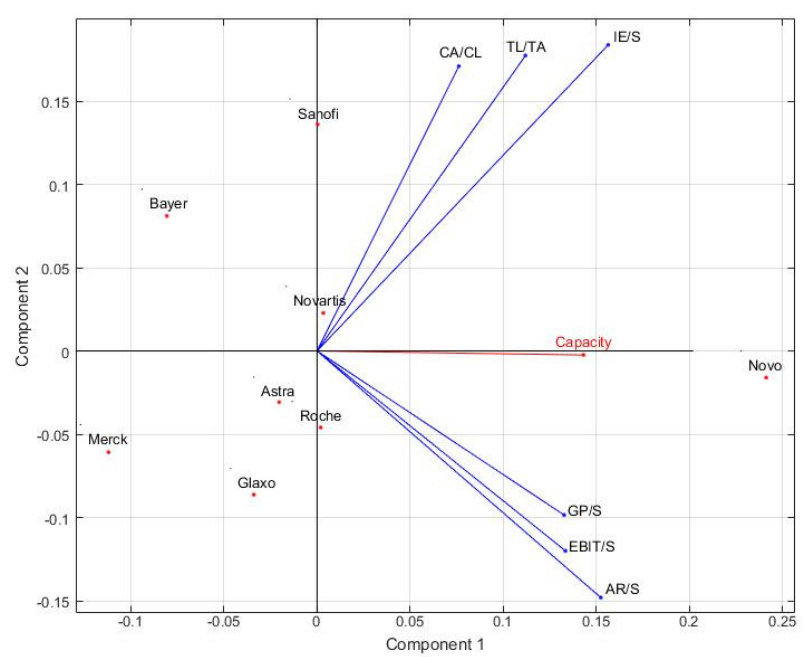

(a) Criteria

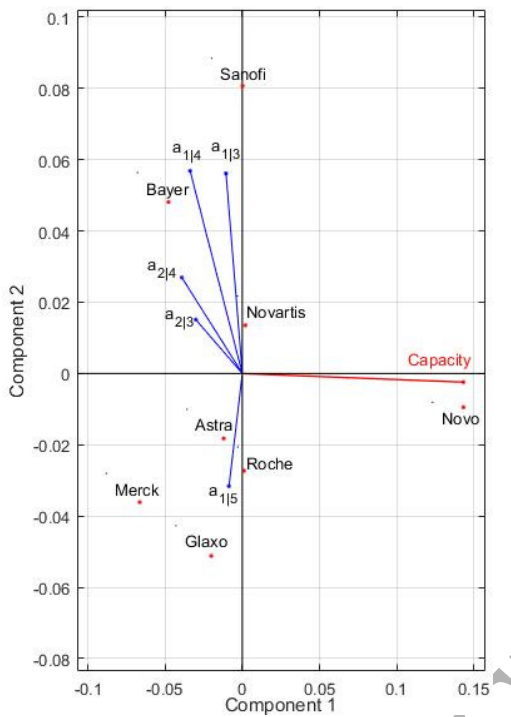

(c) Antagonistic effects

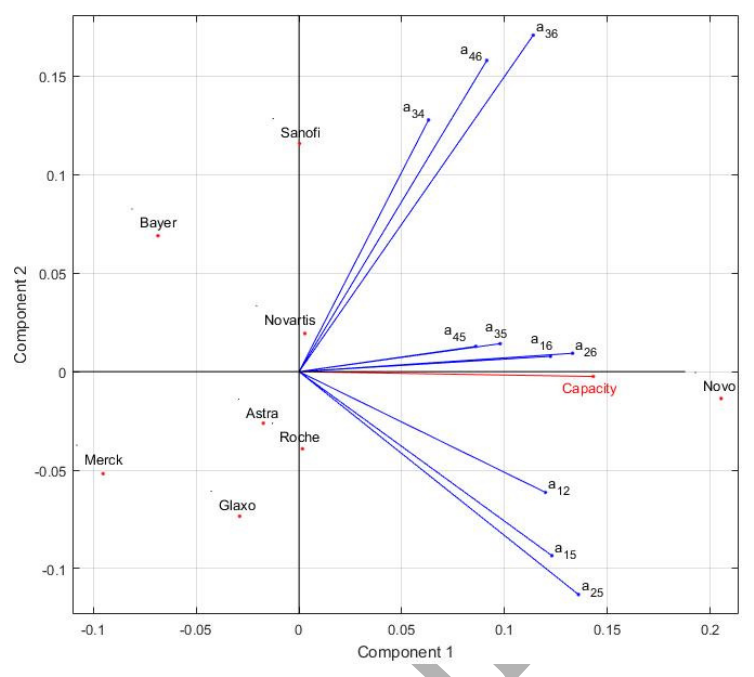

(b) Interactions

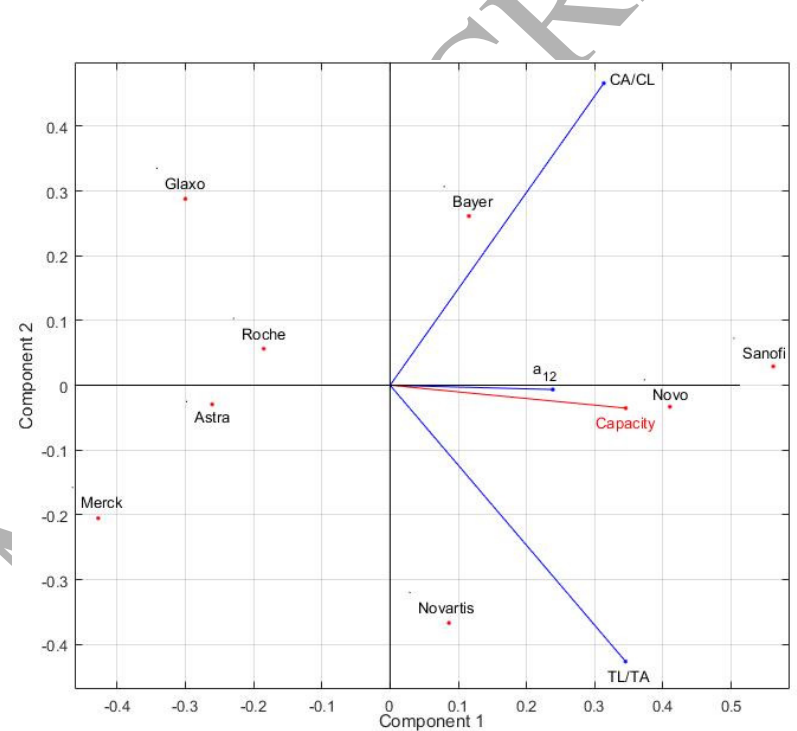

(d) Solvency and Liquidity

Figure 3: Extension of the GAIA plane to take into account interactions and antagonistic effects between criteria

the advantages coming from the capabilities of the new methodology are in part balanced from its complexity related, in particular, to the elicitation of the involved parameters. As already explained, the hierarchical bipolar PROMETHEE methods are based on a bicapacity defined on $\mathcal{P}\left(\mathrm{G}_{\mathrm{E}}\right)$. This means that values for the importance of each elementary criterion $a_{\mathbf{t}}$, the interaction coefficients $a_{\mathbf{t}_{1} \mathbf{t}_{2}}$ for each couple of elementary criteria $\left\{\mathbf{t}_{1}, \mathbf{t}_{2}\right\}$ and the antagonistic effects $a_{\mathbf{t}_{1} \mid \mathbf{t}_{2}}$ for each pair of elementary criteria $\left(\mathbf{t}_{1}, \mathbf{t}_{2}\right)\left(\mathbf{t}_{1}, \mathbf{t}_{2} \in \mathrm{E}_{\mathrm{G}}\right)$ have to be provided. Therefore, the total number of parameters necessary for the application of the methodology is equal to $\left|\mathrm{E}_{\mathrm{G}}\right|+\left(\begin{array}{c}\left|\mathrm{E}_{\mathrm{G}}\right| \\ 2\end{array}\right)+2 D_{\left|\mathrm{E}_{\mathrm{G}}\right|, 2}$. For example, in the previously considered case, being $\left|\mathrm{E}_{\mathrm{G}}\right|=6$, the number of parameters is equal to $6+\left(\begin{array}{l}6 \\ 2\end{array}\right)+D_{6,2}=6+15+30=51$. Of course, asking the DM to provide values for all these parameters is unreasonable.

To solve this issue the use of an indirect technique is required [32], meaning that the DM is asked to provide some preference information in terms of comparisons between alternatives or criteria, interaction between criteria and antagonistic effects between them from which parameters compatible with this information can be inferred. In the following, according to [35], we distinguish between local 
and global preferences at different levels of the hierarchy. A preference, as well as an indifference or an incomparability between alternatives $a, b \in A$ is called local if it is based on considerations of the relation between $a$ and $b$ in terms of $C h_{\mathbf{r}}^{B}\left(P^{B}(a, b), \hat{\mu}\right), C h_{\mathbf{r}}^{B+}\left(P^{B}(a, b), \hat{\mu}\right)$ and $C h_{\mathbf{r}}^{B-}\left(P^{B}(a, b), \hat{\mu}\right)$ for $g_{\mathbf{r}} \in G \backslash \mathrm{G}_{\mathrm{E}}$. Instead, a preference, as well as an indifference or an incomparability between $a$ and $b$ is called global if it is based on consideration of the relation between $a$ and all the others alternatives as well as $b$ and all the others alternatives in terms of $\phi_{\mathbf{r}}^{B}(a), \phi_{\mathbf{r}}^{B+}(a), \phi_{\mathbf{r}}^{B-}(a), \phi_{\mathbf{r}}^{B}(b), \phi_{\mathbf{r}}^{B+}(b)$ and $\phi_{\mathbf{r}}^{B-}(b)$ for $g_{\mathbf{r}} \in G \backslash \mathrm{G}_{\mathrm{E}}$.

The DM can therefore express:

- Preferences on alternatives:

1. $a$ is locally preferred to $b$, translated into $C h^{B}\left(P^{B}(a, b), \hat{\mu}\right)>0$,

2. $a$ is locally preferred to $b$ on $g_{\mathbf{r}}$, translated into $C h^{B}\left(P_{\mathbf{r}}^{B}(a, b), \hat{\mu}\right)>0$,

3. $a$ is comprehensively preferred to $b$, translated into

$-\phi^{B}(a)>\phi^{B}(b)$ if the bipolar PROMETHEE II is considered,

$-\left\{\begin{array}{l}\phi^{B+}(a) \geq \phi^{B+}(b), \\ \phi^{B-}(a) \leq \phi^{B-}(b), \text { if the bipolar PROMETHEE I is considered, } \\ \phi^{B}(a)>\phi^{B}(b)\end{array}\right.$

4. $a$ is comprehensively preferred to $b$ on $g_{\mathbf{r}}$, translated into

$-\phi_{\mathbf{r}}^{B}(a)>\phi_{\mathbf{r}}^{B}(b)$ if the hierarchical bipolar PROMETHEE II is considered,

$-\left\{\begin{array}{l}\phi_{\mathbf{r}}^{B+}(a) \geq \phi_{\mathbf{r}}^{B+}(b), \\ \phi_{\mathbf{r}}^{B-}(a) \leq \phi_{\mathbf{r}}^{B-}(b), \quad \text { if the hierarchical bipolar PROMETHEE I is considered, } \\ \phi_{\mathbf{r}}^{B}(a)>\phi_{\mathbf{r}}^{B}(b)\end{array}\right.$

5. $a$ is locally indifferent to $b$, translated into the constraint $C h^{B}\left(P^{B}(a, b), \hat{\mu}\right)=0$,

6. $a$ is locally indifferent to $b$ on $g_{\mathbf{r}}$, translated into the constraint $C h^{B}\left(P_{\mathbf{r}}^{B}(a, b), \hat{\mu}\right)=0$,

7. $a$ is comprehensively indifferent to $b$, translated into the constraints

- $\phi_{\mathbf{r}}^{B}(a)=\phi_{\mathbf{r}}^{B}(b)$ if the hierarchical bipolar PROMETHEE II is considered,

$-\left\{\begin{array}{l}\phi_{\mathbf{r}}^{B+}(a)=\phi_{\mathbf{r}}^{B+}(b), \\ \phi_{\mathbf{r}}^{B-}(a)=\phi_{\mathbf{r}}^{B-}(b)\end{array}\right.$ if the hierarchical bipolar PROMETHEE I is considered,

- Preferences on importance of and interactions between elementary criteria:

- the importance of criterion $g_{\mathbf{t}}$ varies between $l_{\mathbf{t}}$ and $u_{\mathbf{t}}$, translated into the constraint $l_{\mathbf{t}} \leq a_{\mathbf{t}} \leq u_{\mathbf{t}}$

- $g_{\mathbf{t}_{1}}$ is more important than $g_{\mathbf{t}_{2}}$, translated into the constraint $a_{\mathbf{t}_{1}}>a_{\mathbf{t}_{2}}$,

$-g_{\mathbf{t}_{1}}$ and $g_{\mathbf{t}_{2}}$ are equally important, translated into the constraint $a_{\mathbf{t}_{1}}=a_{\mathbf{t}_{2}}$,

- $g_{\mathbf{t}_{1}}$ and $g_{\mathbf{t}_{2}}$ are positively interacting, translated into the constraint $a_{\mathbf{t}_{1} \mathbf{t}_{2}}>0$,

$-g_{\mathbf{t}_{1}}$ and $g_{\mathbf{t}_{2}}$ are negatively interacting, translated into the constraint $a_{\mathbf{t}_{1} \mathbf{t}_{2}}<0$,

$-g_{\mathbf{t}_{2}}$ exercises an antagonistic effect over $g_{\mathbf{t}_{1}}$, translated into the constraint $a_{\mathbf{t}_{1} \mid \mathbf{t}_{2}}^{+}<0$.

- Preferences on importance of and interactions between non-elementary criteria:

- $g_{\mathbf{r}_{1}}$ is more important than $g_{\mathbf{r}_{2}}$, translated into the constraint

$$
\sum_{\mathbf{t}_{1} \in \mathrm{E}\left(g_{\mathbf{r}_{1}}\right)} a_{\mathbf{t}_{1}}>\sum_{\mathbf{t}_{2} \in \mathrm{E}\left(g_{\mathbf{r}_{2}}\right)} a_{\mathbf{t}_{2}}
$$


$-g_{\mathbf{r}_{1}}$ and $g_{\mathbf{r}_{2}}$ are positively (negatively) interacting, translated into the constraint

$$
\sum_{\substack{\mathbf{t}_{1} \in \mathrm{E}\left(g_{\mathbf{r}_{1}}\right) \\ \mathbf{t}_{2} \in \mathrm{E}\left(g_{\mathbf{r}_{2}}\right)}} a_{\mathbf{t}_{1} \mathbf{t}_{2}}>0 \quad(<0) .
$$

In this case, the DM could be willing to be more precise in his preferences, specifying the nature of the interactions between some $g_{\mathbf{t}_{1}}, \mathbf{t}_{1} \in \mathbf{E}\left(g_{\mathbf{r}_{1}}\right)$ and some $g_{\mathbf{t}_{2}}, \mathbf{t}_{2} \in \mathbf{E}\left(g_{\mathbf{r}_{2}}\right)$; this means that $a_{\mathbf{t}_{1} \mathbf{t}_{2}}$ can be greater than zero for some $\mathbf{t}_{1} \in \mathrm{E}\left(g_{\mathbf{r}_{1}}\right)$ and $\mathbf{t}_{2} \in \mathrm{E}\left(g_{\mathbf{r}_{2}}\right)$, while $a_{\mathbf{t}_{1} \mathbf{t}_{2}}$ can be lower than zero for some $\mathbf{t}_{1} \in \mathbf{E}\left(g_{\mathbf{r}_{1}}\right)$ and $\mathbf{t}_{2} \in \mathbf{E}\left(g_{\mathbf{r}_{2}}\right)$;

- criterion $g_{\mathbf{r}_{2}}$ exercises an antagonistic effect over $g_{\mathbf{r}_{1}}$, translated into the constraint:

$$
\sum_{\substack{\mathbf{t}_{1} \in E\left(g_{\mathbf{r}_{1}}\right) \\ \mathbf{t}_{2} \in \mathrm{E}\left(g_{\mathbf{r}_{2}}\right)}} a_{\mathbf{t}_{1} \mid \mathbf{t}_{2}}<0 .
$$

As in the previous case, the DM can be willing to specify if there is some antagonist effect exercised from elementary criteria descending from $g_{\mathbf{r}_{2}}$ over elementary criteria descending from $g_{\mathbf{r}_{1}}$. This means that $a_{\mathbf{t}_{1} \mid \mathbf{t}_{2}}<0$ for some $\mathbf{t}_{1} \in \mathrm{E}\left(g_{\mathbf{r}_{1}}\right)$ and $\mathbf{t}_{2} \in \mathrm{E}\left(g_{\mathbf{r}_{2}}\right)$.

Let us observe that because of the symmetry conditions (13), $a_{\mathbf{t}}^{+} \geqslant a_{\mathbf{t}}^{-}=a_{\mathbf{t}}$ and $a_{\mathbf{t}_{1} \mathbf{t}_{2}}^{+}=a_{\mathbf{t}_{1} \mathbf{t}_{2}}^{-}=$ $a_{\mathbf{t}_{1} \mathbf{t}_{2}}$ for all $\mathbf{t}, \mathbf{t}_{1}, \mathbf{t}_{2} \in \mathrm{E}_{\mathrm{G}}$. Consequently, in the above constraints, we used $a_{\mathbf{t}}$ and $a_{\mathbf{t}_{1} \mathbf{t}_{2}}$ only, without distinguishing between the positive and the negative importance of criteria and interaction coefficients. Moreover, monotonicity $[M C]$ and normalization $[N C]$ constraints can be written as follows:

$$
[M C]\left\{\begin{array}{c}
a_{\mathbf{t}}+\sum_{\mathbf{t}_{1} \in C} a_{\mathbf{t t}_{1}}+\sum_{\mathbf{t}_{1} \in D} a_{\mathbf{t}_{\mid} \mathbf{t}_{1}}^{+} \geq 0, \quad \forall \mathbf{t} \in \mathrm{E}_{\mathrm{G}}, \forall(C \cup\{\mathbf{t}\}, D) \in \mathcal{P}\left(\mathrm{G}_{\mathrm{E}}\right), \\
a_{\mathbf{t}}+\sum_{\mathbf{t}_{1} \in D} a_{\mathbf{t t}_{1}}+\sum_{\mathbf{t}_{1} \in C} a_{\mathbf{t}_{1} \mid \mathbf{t}}^{+} \geq 0, \forall \mathbf{t} \in \mathrm{E}_{\mathrm{G}}, \forall(C, D \cup\{\mathbf{t}\}) \in \mathcal{P}\left(\mathrm{G}_{\mathrm{E}}\right), \\
{[N C] \sum_{\mathbf{t} \in \mathrm{E}_{\mathrm{G}}} a_{\mathbf{t}}+\sum_{\mathbf{t}_{1} \mathbf{t}_{2} \in \mathrm{E}_{\mathrm{G}}} a_{\mathbf{t}_{1} \mathbf{t}_{2}}=1 .}
\end{array}\right.
$$

In the following, by $E^{D M}$ we shall denote the set of constraints translating the preferences provided by the DM, together with the normalization $[N C]$ and monotonicity $[M C]$ constraints, where strict inequalities are converted into weak inequalities by using an auxiliary variable $\varepsilon$ assumed to be a small value greater than zero (for example, $C h^{B}\left(P^{B}(a, b), \hat{\mu}\right)>0$ is converted into $C h^{B}\left(P^{B}(a, b), \hat{\mu}\right) \geq \varepsilon$ ). If $E^{D M}$ is feasible and $\varepsilon^{*}>0$, where $\varepsilon^{*}=\max \varepsilon$ subject to $E^{D M}$, then there is at least one set of parameters compatible with the preferences provided by the DM. In the opposite case, in which $E^{D M}$ is infeasible or $\varepsilon^{*} \leq 0$, one can check the cause of the infeasibility by using some of the methodologies presented in [43].

In general, if there exists one set of compatible parameters, then there exists an infinity of them. Consequently, the choice of only one of these sets of parameters can be considered arbitary. The Stochastic Multicriteria Acceptability (SMAA) and the Robust Ordinal Regression (ROR), that we shall present in the following sections, avoid this choice by considering simultaneously all compatible sets of parameters.

\subsection{Stochastic Multicriteria Acceptability Analysis}

Stochastic Multicriteria Acceptability Analysis (SMAA) [38] is a family of methods for aiding multicriteria decision making in problems with uncertain, imprecise, or partially missing information. Depending on the model considered for representing the preferences of the DM, different SMAA 
methods have been proposed over the years (see [39] and [47] for two surveys on the SMAA methods). SMAA methods explore the space of parameters compatible with the preferences provided by the DM and defined by the set of constraints $E^{D M}$ as well as the space of the evaluations of the alternatives on the considered criteria in an iterative way ${ }^{6}$. In this case, we shall assume that the evaluations of the alternatives are fixed. Several compatible sets of parameters can be sampled from the corresponding space and, since the set of constraints $E^{D M}$ is linear, one can use the Hit-And-Run algorithm to sample them [45, 48, 49]. For each of these compatible sets of parameters, a different ranking of the considered alternatives can be obtained by the hierarchical bipolar PROMETHEE II method. Analogously, one preference, one indifference and one incomparability relation between the same alternatives can be obtained in case the hierarchical bipolar PROMETHEE I method is applied. For this reason, the application of SMAA in this context can provide the following indices

- the rank acceptability index $b_{\mathbf{r}}^{k}(a)$ (in case one applies the hierarchical bipolar PROMETHEE II method) giving the frequency with which an alternative $a$ gets the $k$-th position in the final ranking computed for criterion $g_{\mathbf{r}}$,

- the preference index $\operatorname{Pre}_{\mathbf{r}}(a, b)$ (both in case of the hierarchical bipolar PROMETHEE I and II methods), called also pairwise winning index in [40], giving the frequency with which $a$ is preferred over $b$ on criterion $g_{\mathbf{r}}$,

- the indifference index $\operatorname{Ind}_{\mathbf{r}}(a, b)$ (both in case of the hierarchical bipolar PROMETHEE I and II methods) giving the frequency with which $a$ and $b$ are indifferent on criterion $g_{\mathbf{r}}$,

- the incomparability index $\operatorname{Inc}_{\mathbf{r}}(a, b)$ (in case of the hierarchical bipolar PROMETHEE I method) giving the frequency with which $a$ and $b$ are incomparable on criterion $g_{\mathbf{r}}$.

\subsubsection{GAIA plane for SMAA}

From the previous Section 4.2, it becomes clear that there is not only one bicapacity $\hat{\mu}$, represented by its corresponding vector $\hat{\mathbf{a}}$, of parameters compatible with the preferences provided by the DM, but a whole set of them. Consequently, the GAIA plane can be extended to permit to convey information about the plurality of bicapacities compatible with the preferences provided by the DM. The idea of considering the whole set of bicapacities corresponds to the imprecise determination of the weight vector for the classical PROMETHEE methods considered in the PROMETHEE VI Sensitivity Tool [8], in which the weight $w_{j}$ of each criterion $g_{j} \in G$ is supposed to be in an interval, that is $w_{j}^{-} \leq w_{j} \leq w_{j}^{+}$. In this way, taking into account the constraint related to the normalization of the weights, that is $w_{1}+\ldots+w_{m}=1$, a polyhedron of feasible weight vector is determined and it is projected on the GAIA plane. This projection, denoted by $\Delta$, has the same interpretation of the PROMETHEE/decision axis, but, more realistically, it takes into account ill-determination and imprecision of the weights. The projection $\Delta$ permits to distinguish between:

- soft problems, when the projection of the set of feasible weight vectors does not include the origin of the axis in the GAIA plane and, then, the direction of the PROMETHEE decision axis is similar for all feasible weight vectors,

- hard problems, when the projection of the set of feasible weight vectors includes the origin of the axis in the GAIA plane and, then, the PROMETHEE decision axis corresponding to feasible weight vectors take all directions.

\footnotetext{
${ }^{6}$ Let us observe that the DM is not obliged to provide any preference information. Just in case he is not willing to provide any preferences in terms of comparison between alternatives or in terms of comparison between criteria and interaction or antagonistic effects between them, then the set of constraints $E^{D M}$ is composed of the monotonicity $[\mathrm{MC}]$ and normalization $[\mathrm{NC}]$ constraints only.
} 
The plurality of feasible weight vectors is also considered by [30] that proposes to couple SMAA method with the GAIA plane. With this aim, on the basis of the results obtained by the random sampling of the preferential parameters, they estimate their distribution that is consequently projected on the GAIA plane.

Here we propose to project directly the vectors $\hat{\mathbf{a}}$, representing the feasible bicapacities $\hat{\mu}$, rather than one estimation of their distribution representing the sampled bicapacities on the GAIA plane, so that the DM can have a useful visualization of the decision problem and of the main results obtained through our extension of the SMAA-PROMETHEE methods. More in detail, on the GAIA plane one can project:

- the whole set of feasible bicapacities $\hat{\mu}$, represented by the corresponding vectors $\hat{\mathbf{a}}$, so that the DM can visualize the set of PROMETHEE decision axis as well as importance, interaction and antagonistic effects, compatible with the preference information,

- the set of feasible bicapacities $\hat{\mu}$ for which an alternative attains a given ranking position (first, second, etc...);

- the set of feasible bicapacities $\hat{\mu}$ for which one alternative is preferred, indifferent or incomparable to another one.

Clearly, the GAIA plane technique can be also applied to single non-elementary criteria in order to obtain a graphical representation of the SMAA results in case of hierarchical structure of the set of criteria. In Section 6, considering the problem of evaluating pharmaceutical companies, we show an application of the GAIA plane technique coupled with SMAA in case of hierarchy of interacting criteria.

\subsection{Robust Ordinal Regression}

Robust Ordinal Regression (ROR; [13, 28]) takes into account not only one but the whole set of parameters compatible with the preference information provided by the DM, by defining a necessary and a possible preference relation. In our context, two different necessary and possible preference relations can be defined for each non-elementary criterion $g_{\mathbf{r}}$. Given alternatives $a$ and $b, a$ is necessarily preferred to $b$ on $g_{\mathbf{r}}$ iff $a$ is at least as good as $b$ on $g_{\mathbf{r}}$ for all compatible models, while $a$ is possibly preferred to $b$ on $g_{\mathbf{r}}$ iff $a$ is at least as good as $b$ on $g_{\mathbf{r}}$ for at least one compatible model.

The previous preference relations can be obtained as follows:

- $a$ is necessarily preferred to $b$ on $g_{\mathbf{r}}$ :

- locally, iff $E^{N}=E^{D M} \cup\left\{C h^{B}\left(P_{\mathbf{r}}^{B}(a, b), \hat{\mu}\right)+\varepsilon \leq 0\right\}$ is infeasible or $\varepsilon_{N}^{*} \leq 0$, where $\varepsilon_{N}^{*}=\max \varepsilon$ subject to $E^{N}$,

- globally and considering the hierarchical bipolar PROMETHEE II method, if $E^{N}=$ $E^{D M} \cup\left\{\phi_{\mathbf{r}}^{B}(a)+\varepsilon \leq \phi_{\mathbf{r}}^{B}(b)\right\}$ is infeasible or $\varepsilon_{N}^{*} \leq 0$, where $\varepsilon_{N}^{*}=\max \varepsilon$ subject to $E^{N}$

- globally and considering the hierarchical bipolar PROMETHEE I method, if $E^{N}$ is infeasible or $\varepsilon_{N}^{*} \leq 0$, where $\varepsilon_{N}^{*}=\max \varepsilon$ subject to $E^{N}$ and

$$
E^{N}=\left\{\begin{array}{l}
E^{D M} \\
\phi_{\mathbf{r}}^{B+}(a)+\varepsilon \leq \phi_{\mathbf{r}}^{B+}(b)+2 M_{1} \text { and } \phi_{\mathbf{r}}^{B-}(a)+2 M_{2} \geq \phi_{\mathbf{r}}^{B-}(b)+\varepsilon \\
\quad \text { where } M_{i} \in\{0,1\}, i=1,2, \text { and } \sum_{i=1}^{2} M_{i} \leq 1
\end{array}\right.
$$

- $a$ is possibly preferred to $b$ on $g_{\mathbf{r}}$ : 
- locally, iff $E^{P}=E^{D M} \cup\left\{C h^{B}\left(P_{\mathbf{r}}^{B}(a, b), \hat{\mu}\right) \geq 0\right\}$ is feasible and $\varepsilon_{P}^{*}>0$, where $\varepsilon_{P}^{*}=\max \varepsilon$ subject to $E^{P}$,

- globally and considering the hierarchical bipolar PROMETHEE II method, if $E^{P}=E^{D M} \cup$ $\left\{\phi_{\mathbf{r}}^{B}(a) \geq \phi_{\mathbf{r}}^{B}(b)\right\}$ is feasible and $\varepsilon_{P}^{*}>0$, where $\varepsilon_{P}^{*}=\max \varepsilon$ subject to $E^{P}$,

- globally and considering the hierarchical bipolar PROMETHEE I method, if $E^{P}$ is feasible and $\varepsilon_{P}^{*}>0$, where $\varepsilon_{P}^{*}=\max \varepsilon$ subject to $E^{P}$ and

$$
E^{P}=\left\{\begin{array}{l}
E^{D M} \\
\phi_{\mathbf{r}}^{B+}(a) \geq \phi_{\mathbf{r}}^{B+}(b), \\
\phi_{\mathbf{r}}^{B-}(a) \leq \phi_{\mathbf{r}}^{B-}(b) .
\end{array}\right.
$$

All the parameters concerning on the interactions which are not considered by the DM are set equal to zero.

\section{The flow chart and main steps of the proposed method}

In this section we shall briefly summarize the main steps inyolved in the application of the hierarchical bipolar PROMETHEE method (see the flow chart in Fig. 4).

Figure 4: Flow chart of the hierarchical bipolar PROMETHEE method
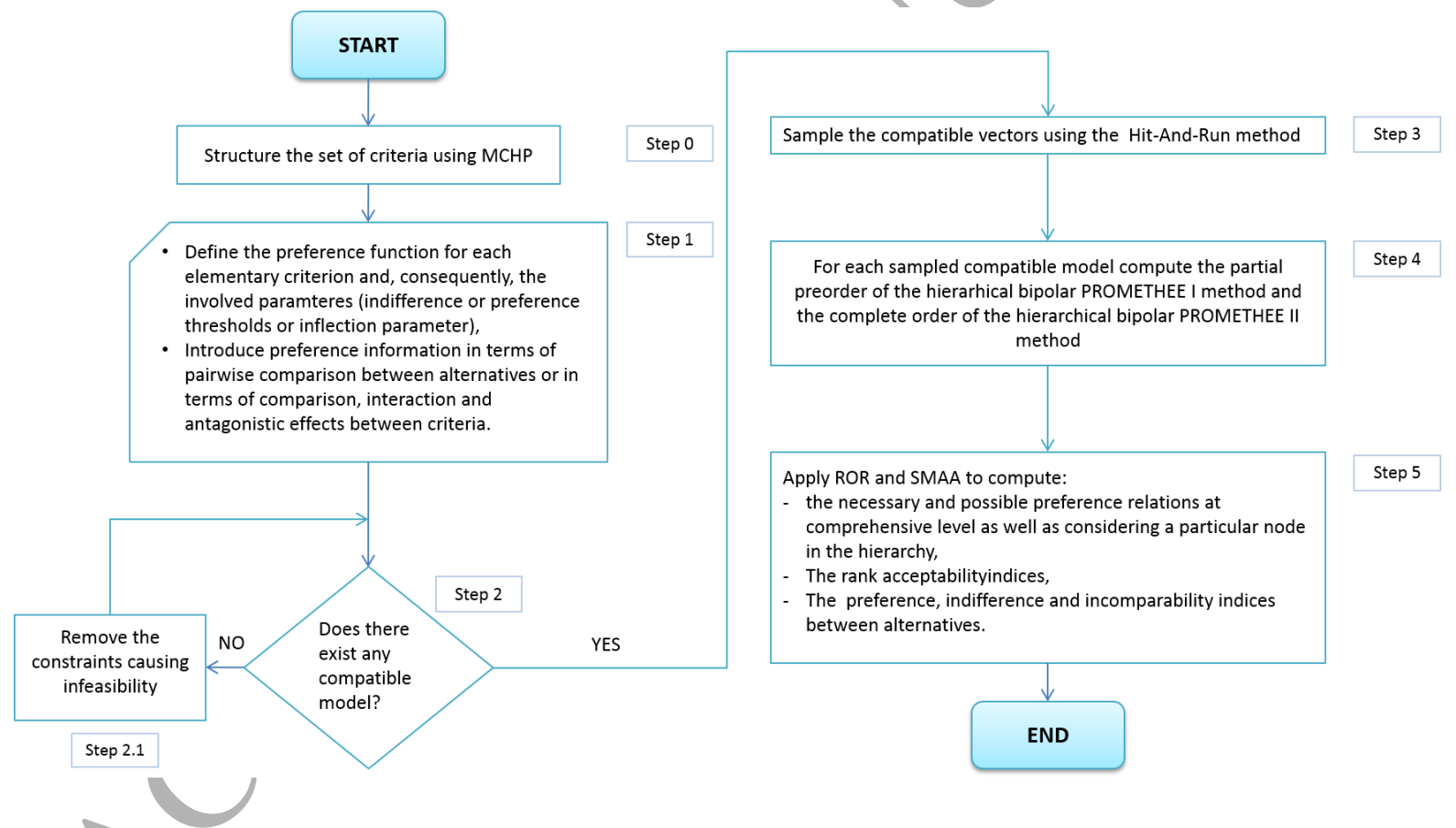

Step 0) The problem is structured in a hierarchical way by defining elementary criteria and all other criteria placed at intermediate level between the root node and the bottom of the hierarchy (Section 2.3);

Step 1) For each elementary criterion one of the six preference functions proposed in [9] has to be chosen. Each of them involves the definition of at most 2 parameters being indifference and preference threshold or the inflection parameter (Section 2.2);

The DM is invited to provide some preference in terms of pairwise comparison between alternatives (preference or indifference between them) at comprehensive level (that is considering all 
alternatives) or at a partial level (considering only two alternatives). Moreover, these preferences could be provided at the root level (considering all elementary criteria at the same time), or at a certain node (considering the elementary criteria descending from a certain criterion only). If he is willing to, the DM can also provide some preference information in terms of importance and comparison between criteria (preference or indifference between them), positive or negative interaction or antagonistic effect between them (Section 4.1);

Step 2) The analyst checks if there exists at least one model compatible with the preferences provided by the DM. If this is not the case, the cause of the infeasibility has to be investigated (Section 4.1);

Step 3) Several sets of parameters compatible with the preferences provided by the DM (compatible models) are randomly sampled by using the Hit-And-Run method (Section 4.2);

Step 4) For each compatible model the bipolar positive, negative and net flows of each alternative are computed at root level as well as at partial level. On the basis of this flow the preference and indifference relations can be computed considering the hierarchical bipolar PROMETHEE I and II methods, while the incomparability relation can be computed in considering the hierarchical bipolar PROMETHEE I method (Section 3);

Step 5) The SMAA (Section 4.2) and ROR (Section 4.3) methodologies are applied to get robust recommendations on the problem at hand and are graphically) represented on the GAIA plane to supply an easily understandable intuition.

This procedure, that has been built for the extension of the PROMETHEE I and II methods to take into account hierarchy and interaction between criteria as well as robustness concerns, can be easily adapted to other PROMETHEE methods (PROMETHEE III, IV, V, VI and GDSS) which extensions have been briefly described in the Appendix.

\section{Didactic example}

In this section we show how to apply the proposed methodology to the financial decision problem handled starting from section 2.3. The eight most capitalized European pharmaceutical companies are evaluated on three aspects: Profitability (P), Solvency and Liquidity (S\&L) and Managerial Performance (MP). The evaluations of the eight considered companies on the six elementary criteria descending from the three macro-aspects have been extracted from [1].

At first, for each pair of alternatives $(a, b) \in A \times A$ the bipolar vector $P^{B}(a, b)$ has been computed as described in section 2.2. After that, since we would like to apply the hierarchical extension of the bipolar PROMETHEE methods, we asked the DM to provide some preference information. He was confident in giving us the following information:

1. comparisons between the importance of criteria:

- $P$ is more important than $S \& L$ that, in turn, is more important than $M P$,

- $\left\{\begin{array}{l}E B I T / S \text { is more important than } G P / S ; \\ G P / S \text { is more important than } I E / S ; \\ I E / S \text { is more important than } T L / T A ; \\ T L / T A \text { is more important than } A R / S\end{array}\right.$

2. positive and negative interactions between criteria:

- $P$ and $M P$ are negatively interacting, 
- $S \& L$ and $M P$ are negatively interacting; in particular, all elementary criteria descending from $S \& L$ are negatively interacting with all elementary criteria descending from $M P$; this means that the pairs of elementary criteria $\{T L / T A, A R / S\},\{T L / T A, I E / S\}$, $\{C A / C L, A R / S\}$ and $\{C A / C L, I E / S\}$ are negatively interacting,

- $\left\{\begin{array}{l}G P / S \text { and } E B I T / S \text { are negatively interacting; } \\ G P / S \text { and } A R / S \text { are positively interacting; } \\ E B I T / S \text { and } I E / S \text { are positively interacting; } \\ T L / T A \text { and } C A / C L \text { are negatively interacting; }\end{array}\right.$

3. antagonistic effect between criteria:

- $S \& L$ has an antagonistic effect over $P$; in particular, all elementary criteria descending from $S \& L$ have an antagonistic effect over all elementary criteria descending from $P$; this means that an antagonistic effect is exercised from $T L / T A$ over $G P / S$ and $E B I T / S$ and from $C A / C L$ over $G P / S$ and $E B I T / S$;

- $A R / S$ has an antagonistic effect over $G P / S$.

At this point, the ROR and the SMAA methodologies are applied to get robust recommendations on the problem at hand. By applying the ROR to the hierarchical bipolar PROMETHEE II method, we get the necessary preference relation at global level as well as on the three considered aspects as shown in Figure 5.

As one can see in Fig. 5(a), Novo Nordisk is comprehensively necessarily preferred to all other companies, while all companies apart from Bayer are comprehensively necessarily preferred to Merck. Considering the remaining six companies, nothing can be concluded looking at this figure since they are incomparable.

Something different can be observed on the three single main aspects:

- with respect to profitability (Fig. 5(b)), again Novo Nordisk is the best, while Merck is the worst one. Anyway, only the incomparabilities between AstraZeneca and Bayer and between Roche and AstraZeneca can be highlighted,

- on solvency and liquidity (Fig. 5(c)) Novo Nordisk is no longer the best company. Instead, Sanofi is necessarily preferred to all other companies but Novartis; looking at the lowest level of the diagram some companies are necessarily preferred to Glaxo, Roche, AstraZeneca and Merck, while each of them is not necessarily preferred to anyone else,

- considering managerial performance (Fig. 5(d)), Novo Nordisk is, again, the best company, while Merck, Glaxo and Bayer are at the bottom of the ranking.

As previously observed, the necessary preference relations computed at the global level as well as with respect to the three considered aspects, leave many pairs of companies incomparable. For example, looking at Fig. 5(c), we can observe that, on one hand, Novartis is not necessarily preferred to any other company and, on the other hand, no company is necessarily preferred to it. For this reason, we decided to apply also the SMAA methodology to the hierarchical bipolar PROMETHEE methods to get some estimate of the frequency with which a company gets a certain rank position or the frequency with which one company is preferred to another not only at global level but also with respect to a particular aspect.

As shown in Tables 10(a) and 10(b), Novo Nordisk is undoubtedly the best among the eight companies since it achieves always the first position in the ranking and, consequently, it is always preferred to all other companies. On the opposite side, the Merck is always in the last position and all companies are always preferred to it. Other information can be gathered: 


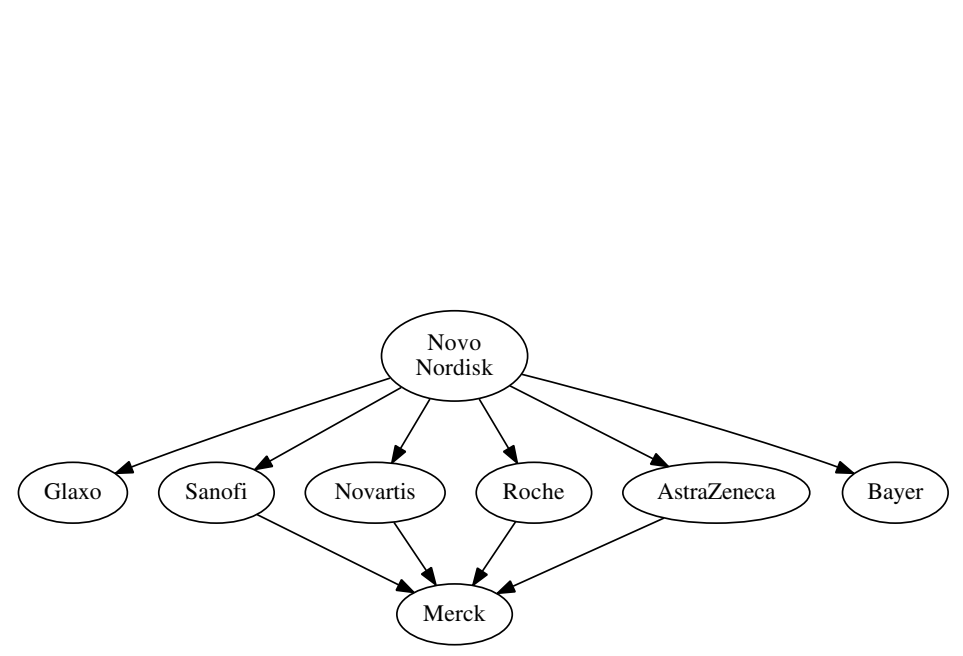

(a) Comprehensive level

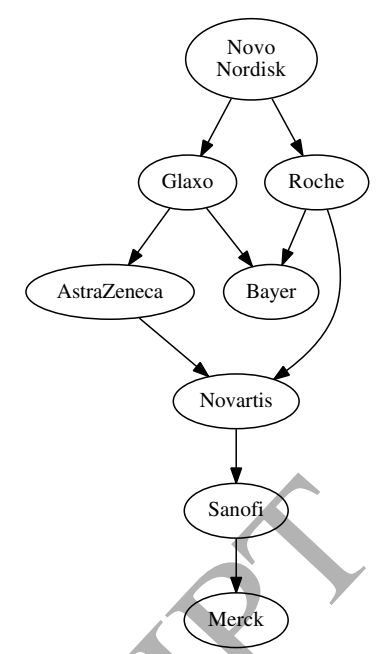

(b) Profitability (P)

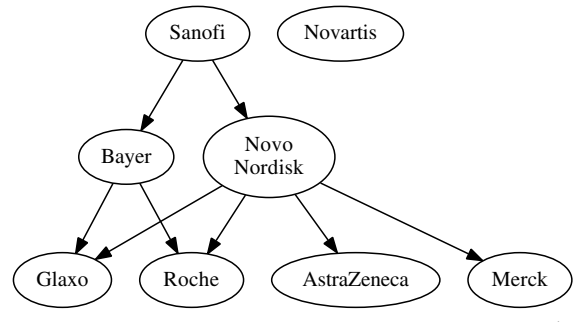

(c) Solvency and Liquidity (S\&L)

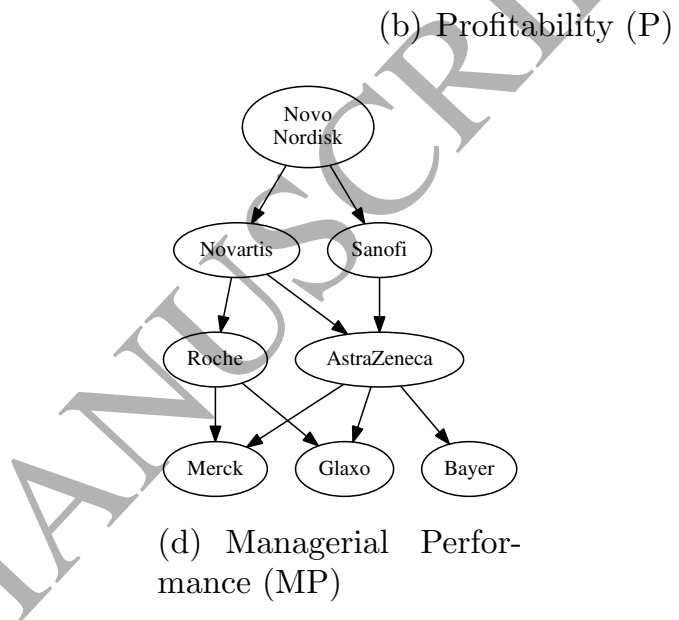

Figure 5: Necessary preference relation at the comprehensive level, as well as with respect to macro-criteria P, S\&L and MP considering the hierarchical bipolar PROMETHEE II method.

- apart from Novo Nordisk and Merck filling always the first and the last position, respectively, all other companies can reach different positions. For instance, Glaxo and Novartis show a nonzero percentage from the third to the seventh position, while the Bayer fills always a position from the fifth to the seventh. Moreover, Roche achieves the second position in $33.25 \%$ of the cases;

- looking at Table 10(b), Sanofi can be considered the second best company since it is preferred to all other companies, apart from Novo Nordisk, with a percentage no lower than $66.75 \%$; conversely, the Bayer is the second worst company since all other ones, apart from Merck, are preferred to it with a percentage no lower than $77.43 \%$;

Similar consíderations can be done also on single aspects. Here we report those related to $S \& L$ :

- while Novo Nordisk was always in the first position at a global level, a different situation can be observed on $S \& L$. Indeed, Sanofi ranks always first (see Table 10(c)) and, consequently, it is always preferred to all other companies (see Table 10(d));

- looking at Table 10(d), most of the pairwise comparisons are certain, while others are not true in all cases. For example, AstraZeneca is preferred to Glaxo with the 85.05\%, while Glaxo is preferred to AstraZeneca with a percentage equal to $14.92 \%$; analogously, Glaxo is preferred to Merck with a percentage of the $92.6 \%$, while the viceversa is true in the $7.4 \%$ of the cases. 
Table 10: Results obtained by applying SMAA to the hierarchical bipolar PROMETHEE II method

(a) Rank acceptability indices (in percentage) at global level

\begin{tabular}{l|cccccccc}
\hline \hline Pharma Company & $\mathbf{b}^{\mathbf{1}}$ & $\mathbf{b}^{\mathbf{2}}$ & $\mathbf{b}^{\mathbf{3}}$ & $\mathbf{b}^{\mathbf{4}}$ & $\mathbf{b}^{\mathbf{5}}$ & $\mathbf{b}^{\mathbf{6}}$ & $\mathbf{b}^{\mathbf{7}}$ & $\mathbf{b}^{\mathbf{8}}$ \\
\hline \hline Glaxo & 0 & 0 & 1.82 & 5.7 & 12.57 & 57.75 & 22.16 & 0 \\
Sanofi & 0 & 66.75 & 31.02 & 2.23 & 0 & 0 & 0 & 0 \\
Novartis & 0 & 0 & 3.79 & 17.07 & 57.88 & 15.85 & 5.41 & 0 \\
Roche Holding & 0 & 33.25 & 62.84 & 3.29 & 0.62 & 0 & 0 & 0 \\
AstraZeneca & 0 & 0 & 0.53 & 71.71 & 27.2 & 0.56 & 0 & 0 \\
Bayer & 0 & 0 & 0 & 0 & 1.73 & 25.84 & 72.43 & 0 \\
Merck & 0 & 0 & 0 & 0 & 0 & 0 & 0 & 100 \\
Novo Nordisk & 100 & 0 & 0 & 0 & 0 & 0 & 0 & 0 \\
\hline \hline
\end{tabular}

(b) Pairwise winning indices (in percentage) at global level

(c) Rank acceptability indices (in percentage) with respect to $\mathrm{S} \& \mathrm{~L}$

\begin{tabular}{l|cccccccc}
\hline \hline Pharma Company & $\mathbf{b}^{\mathbf{1}}$ & $\mathbf{b}^{\mathbf{2}}$ & $\mathbf{b}^{\mathbf{3}}$ & $\mathbf{b}^{\mathbf{4}}$ & $\mathbf{b}^{\mathbf{5}}$ & $\mathbf{b}^{\mathbf{6}}$ & $\mathbf{b}^{\mathbf{7}}$ & $\mathbf{b}^{\mathbf{8}}$ \\
\hline \hline Glaxo & 0 & 0 & 0 & 0 & 1.75 & 13.17 & 77.68 & 7.4 \\
Sanofi & 100 & 0 & 0 & 0 & 0 & 0 & 0 & 0 \\
Novartis & 0 & 0 & 13.92 & 78.62 & 5.71 & 1.75 & 0 & 0 \\
Roche Holding & 0 & 0 & 0 & 7.46 & 92.54 & 0 & 0 & 0 \\
AstraZeneca & 0 & 0 & 0 & 0 & 0 & 85.08 & 14.92 & 0 \\
Bayer & 0 & 0 & 86.08 & 13.92 & 0 & 0 & 0 & 0 \\
Merck & 0 & 0 & 0 & 0 & 0 & 0 & 7.4 & 92.6 \\
Novo Nordisk & 0 & 100 & 0 & 0 & 0 & 0 & 0 & 0 \\
\hline \hline
\end{tabular}

\begin{tabular}{l|cccccccc}
\hline \hline Pharma Company & Gl & Sa & No & Ro & AsZe & Ba & Me & NoNo \\
\hline \hline Glaxo (Gl) & 0 & 1.82 & 19.94 & 0 & 8.08 & 77.43 & 100 & 0 \\
Sanofi (Sa) & 98.18 & 0 & 100 & 66.75 & 99.59 & 100 & 100 & 0 \\
Novartis (No) & 80.06 & 0 & 0 & 3.79 & 20.86 & 93.27 & 100 & 0 \\
Roche (Ro) & 100 & 33.25 & 96.21 & 0 & 99.26 & 100 & 100 & 0 \\
AstraZeneca (AsZe) & 91.92 & 0.41 & 79.14 & 0.74 & 0 & 100 & 100 & 0 \\
Bayer (Ba) & 22.57 & 0 & 6.73 & 0 & 0 & 0 & 100 & 0 \\
Merck (Me) & 0 & 0 & 0 & 0 & 0 & 0 & 0 & 0 \\
Novo Nordisk (NoNo) & 100 & 100 & 100 & 100 & 100 & 100 & 100 & 0 \\
\hline \hline
\end{tabular}

(d) Pairwise winning indices (in percentage) on S\&L

\begin{tabular}{l|cccccccc}
\hline \hline Pharma Company & Gl & Sa & No & Ro & AsZe & Ba & Mé & NoNo \\
\hline \hline Glaxo (Gl) & 0 & 0 & 1.75 & 0 & 14.92 & 0 & 92.6 & 0 \\
Sanofi (Sa) & 100 & 0 & 100 & 100 & 100 & 100 & 100 & 100 \\
Novartis (No) & 98.25 & 0 & 0 & 92.54 & 100 & 13.92 & 100 & 0 \\
Roche (Ro) & 100 & 0 & 7.46 & 0 & 100 & 0 & 100 & 0 \\
AstraZeneca (AsZe) & 85.08 & 0 & 0 & 0 & 0 & 0 & 100 & 0 \\
Bayer (Ba) & 100 & 0 & 86.08 & 100 & 100 & 0 & 100 & 0 \\
Merck (Me) & 7.4 & 0 & 0 & 0 & 0 & 0 & 0 & 0 \\
Novo Nordisk (NoNo) & 100 & 0 & 100 & 100 & 100 & 100 & 100 & 0 \\
\hline \hline
\end{tabular}

All other results obtained by the application of the ROR and SMAA to the hierarchical bipolar PROMETHEE method not only at global level but also with respect to the three particular aspects can be downloaded clicking on the following link: data.

Let us show how the GAIA plane technique can support the DM in the interpretation of all the results supplied by the SMAA-PROMETHEE method. With this aim we present and comment Figures 6(a)-6(c). Let us start by pointing out that Figures 6(a) and 6(b) have the same explained variance $\delta=78.72 \%$ of Figures 3(a),3(b) and 3(c), because they refer to the same matrix $\Phi$. Analogously, Figure $6(\mathrm{c})$ has the same explained variance $\delta=92.47 \%$ of Figure $3(\mathrm{~d})$. Figure $6(\mathrm{a})$ shows the projection on the GAIA plane of a random sample of 10,000 compatible bicapacities. It is presented also the PROMETHEE decision axis corresponding to the barycenter of the sampled compatible bicapacities. Looking at Figure 6(a) it is evident how much robust is the best position of Novo Nordisk, that is in the same direction of the projections of all the compatible bicapacities, as well as the last position of Merck, which is in the opposite direction. Figure 6(b), instead, permits to have a visualization of the $33.25 \%$ of compatible bicapacities for which, according to Table 10(a), Roche Holding gets the second rank position. Comparing them with the whole set of bicapacities represented in Figure 6(a), one can see that Roche Holding is the second for those capacities giving more importance to criteria GP/S, EBIT/S and AR/S. Observe that, again on the basis of Table 10(a), for the other compatible bicapacities Sanofi attains the second rank position. Consequently, the compatible bicapacities shown in 6(a) but not in 6(b) are those for which the second best company is Sanofi, which is therefore preferred to Roche Holding for those bicapacities giving more importance to criteria CA/CL, TL/TA and/E/S. Finally, Figure 6(c) presents the projection of the $85.08 \%$ compatible bicapacities for which, aecording to Table 10(d), AstraZeneca is preferred to Glaxo with respect to Solvency and Liquidity. Of course, all the rich and varied information supplied by PROMETHEE method with respect to all criteria in the hierarchy can be visualized analogously, permitting to the DM to gain a better insight of the decision problem and of the recommendation obtained through the decision process.

To conclude this section, we would like to show the usefulness of considering the MCHP in the problem at hand. To this aim, in Fig. 7 we have shown the comparison between Sanofi and Novartis in terms of frequency of preference of one over the other. In particular, Sanofi is always preferred to 


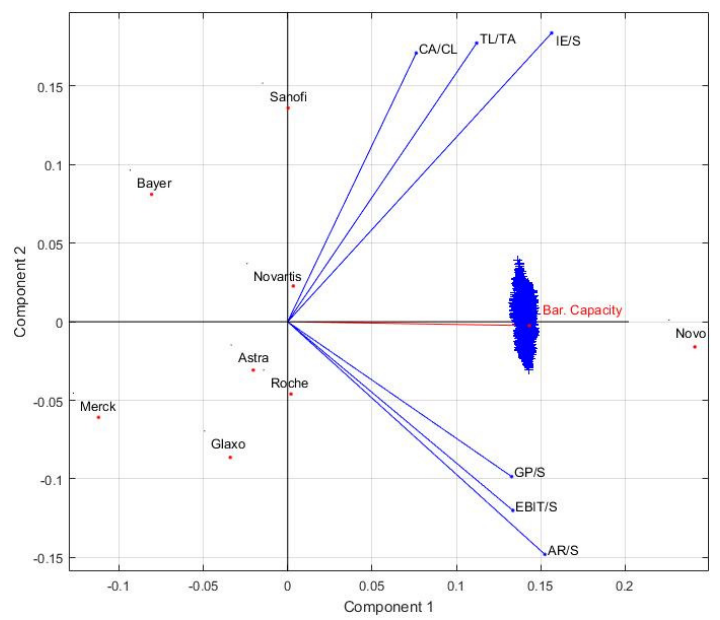

(a) Sampling of 10,000 compatible capacities

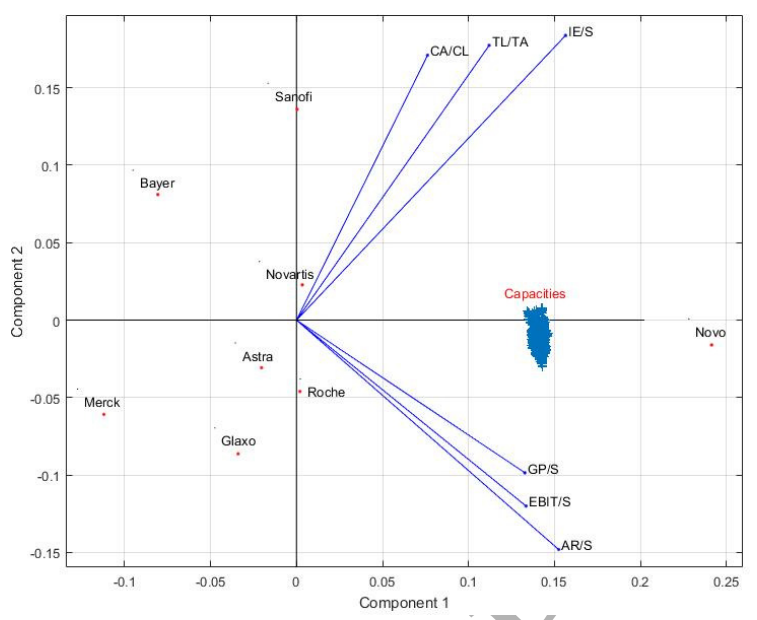

(b) Compatible capacities for which Roche Holding achieves the second position at global level

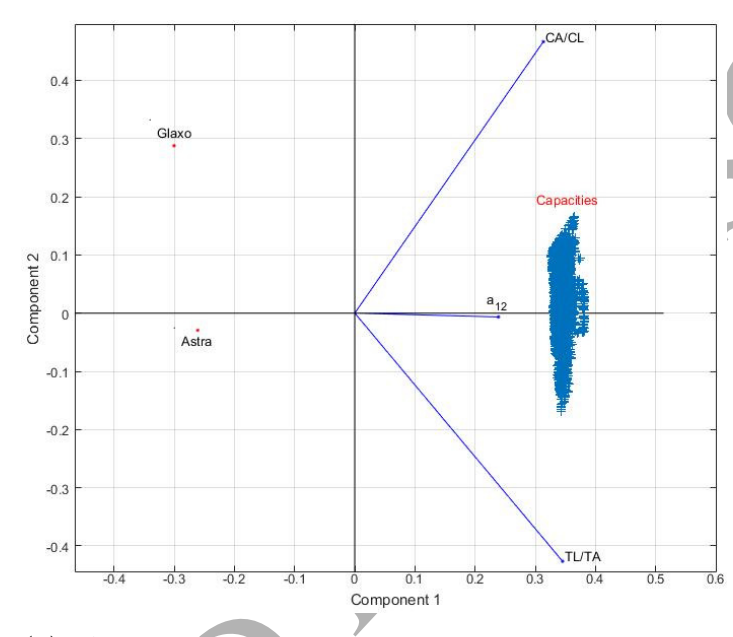

(c) A sampling of compatible capacities for which AstraZeneca is preferred to Glaxo with respect to Solvency and Liquidity

Figure 6: GAIA plane for SMAA-PROMETHEE applied to the hierarchy of interacting criteria

Novartis both at global level as well as on $S \& L$, while Novartis is always preferred to Sanofi on $P$. A not so certain comparison can be done on $M P$. Indeed, on one hand, Sanofi is preferred to Novartis with a frequency of $58.39 \%$, while the viceversa is true in the remaining $41.61 \%$. Let us observe that so detailed considerations can be possible only using the extension of the bipolar PROMETHEE method to the case of criteria structured in a hierarchical way.

\section{Conclusions}

Real world decision problems generally present the following features: criteria structured in a hierarchical way, positive, negative and antagonist effects between considered criteria and uncertainty or imprecision in the definition of the parameters as well as on the evaluations of the alternatives at hand. PROMETHEEE methods are widely used in Multiple Criteria Decision Aiding (MCDA) [6] to deal with such type of problems. Even if some extensions of their basic version take into account these aspects singularly, to the best of our knowledge, does not exist any PROMETHEE method considering simultaneously all of them. For this reason, in order to fill this gap, in this work we have proposed an extension of the PROMETHEE methods. Even if other methods based on 
Figure 7: Frequency of preference of Novartis and Sanofi and viceversa at global level as well as on the three main aspects considering the hierarhical bipolar PROMETHEE II method

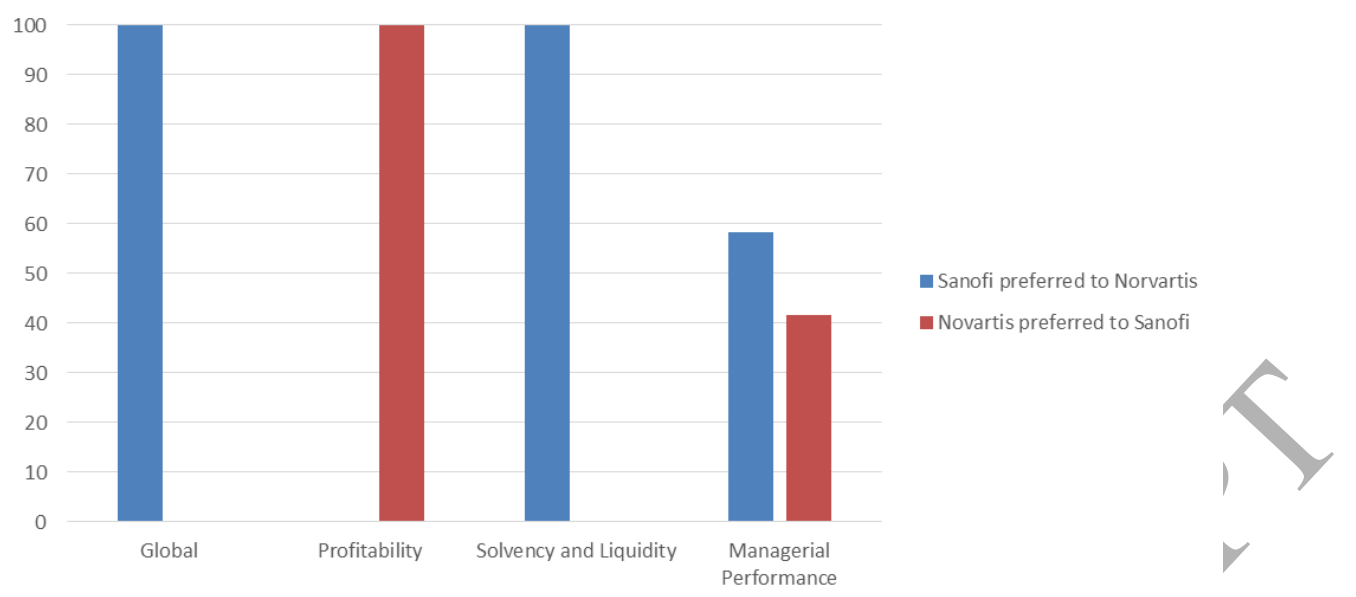

ELECTRE [44] and Multiple Attrubute Value Theory (MAVT) [36] methods have been developed to take into account all the mentioned aspects simultaneously (see $[3,17])$, the results obtained by the new proposed methodology are different from those obtained by the others. This difference is related to the construction of the indifference, preference or incomparability relations which are obtained by means of an exploitation procedure based on the positive, negative and net flows that are computed comparing each alternative with all the others. In MAVT, the indifference and preference relations (the incomparability relation can not be defined in using value functions) are instead built by comparing pairwise the alternatives neglecting all other alternatives, while in ELECTRE methods the exploitation procedures are based on different concepts (kernel and distillation). The simultaneous comparison of each alternative with all the others is therefore an added value of the new proposal.

The new hierarchical bipolar PROMETHEE methods combine the Multiple Criteria Hierarchy Process (MCHP), the PROMETHEE method and the bipolar Choquet integral together with the Robust Ordinal Regression (ROR) and the Stochastic Multicriteria Acceptability Analysis (SMAA). The MCHP is a methodology recently proposed in literature to consider problems characterized by criteria structured in an hierarchy. It permits to analyze the problem at hand not only at global level but also considering single aspects. The bipolar Choquet integral considers the possible interactions and antagonist effects between criteria. In particular, we proposed for the first time an extension of the bipolar Choquet integral to problems presenting criteria organized in an hierarchy. Finally, considering indirect preference information provided by the Decision Maker (DM), ROR and SMAA methodologies proyide robust recommendations with respect to the problem at hand by exploring the whole set of parameters compatible with the provided preferences. All this decision procedure is supported by the graphical visualization supplied by an extension of the GAIA plane technique that permits to take into account interaction of criteria organized in a hierarchy as well as the plurality of feasible parameters compatible with the provided preference information.

We underlined two drawbacks of the proposed methodology. One is related to the huge number of parameters that need to be inferred to apply the method and another regards the consideration of precise evaluations instead of imprecise and uncertain ones. With respect to the first point, as mentioned above, we proposed to use an indirect preference information and, in particular, the ROR and SMAA methodologies as well as the use of a GAIA plane visualization. The second point can be instead considered as a further extension of the proposed methodology in which imprecise and uncertain evaluations can be taken into account by using, for example, fuzzy numbers as done in [20]. Regarding the consideration of uncertainty in MCDA, please see also [7, 10, 31, 41].

We would like to underline that the proposed method is not a simple aggregation of methods 
already existing. The hierarchical bipolar PROMETHEE method is the result of a harmonization of all of them involving a fine adaptation. Moreover we believe that the points we considered are crucially relevant in many real world decision problems that can benefit from the methodology we have proposed.

\section{Acknowledgments}

The authors would like to thank the two anonymous Reviewers whose comments have helped to considerably improve this manuscript. Moreover, the authors wish to acknowledge the funding by the FIR of the University of Catania "BCAEA3, New developments in Multiple Criteria Decision Aiding (MCDA) and their application to territorial competitiveness" and by the research project "Data analytics for entrepreneurial ecosystms, sustainable development and wellbeing indices" of the Department of Economics and Business of the University of Catania. The third author has also benefited of the fund "Chance" of the University of Catania.

\section{References}

\section{References}

[1] Thomson-reuters dataset. http://thomsonreuters.com/en.html.

[2] H. Abdi and L.J. Williams. Principal component analysis. Wiley interdisciplinary reviews: computational statistics, 2(4):433-459, 2010.

[3] S. Angilella, S. Corrente, S. Greco, and R. Słowiński. Robust Ordinal Regression and Stochastic Multiobjective Acceptability Analysis in multiple criteria hierarchy process for the Choquet integral preference model. Omega, (63):154-169, 2016.

[4] M. Bagherikahvarin and Y. De Smet. A ranking method based on DEA and PROMETHEE II (a rank based on DEA \& PR. II). Measurement, 89:333-342, 2016.

[5] M. Bagherikahvarin and Y. De Smet. Determining new possible weight values in PROMETHEE: a procedure based on data envelopment analysis. Journal of the Operational Research Society, 68(5):484-495, 2017.

[6] M. Behzadian, R.B. Kazemzadeh, A. Albadvi, and M. Aghdasi. PROMETHEE: A comprehensive literature review on methodologies and applications. European journal of Operational research, 200(1):198<215, 2010.

[7] M.J. Beynon and P. Wells. The lean improvement of the chemical emissions of motor vehicles based on preference ranking: A PROMETHEE uncertainty analysis. Omega, 36(3):384-394, 2008 .

[8] J.P. Brans and B. Mareschal. The PROMETHEE VI procedure: how to differentiate hard from soft multicriteria problems. Journal of Decision Systems, 4(3):213-223, 1995.

[9] J.P. Brans and Ph. Vincke. A preference ranking organisation method: The PROMETHEE method for MCDM. Management Science, 31(6):647-656, 1985.

[10] C.A.V. Cavalcante and A.T. De Almeida. A multi-criteria decision-aiding model using PROMETHEE III for preventive maintenance planning under uncertain conditions. Journal of Quality in Maintenance Engineering, 13(4):385-397, 2007. 
[11] Y.-H. Chen, T.-C. Wang, and C.-Y. Wu. Strategic decisions using the fuzzy PROMETHEE for IS outsourcing. Expert Systems with Applications, 38(10):13216-13222, 2011.

[12] S. Corrente, S. Greco, and R. Słowiński. Multiple Criteria Hierarchy Process in Robust Ordinal Regression. Decision Support Systems, 53(3):660-674, 2012.

[13] S. Corrente, S. Greco, M. Kadziński, and R. Słowiński. Robust ordinal regression in preference learning and ranking. Machine Learning, 93:381-422, 2013.

[14] S. Corrente, S. Greco, and R. Słowiński. Multiple Criteria Hierarchy Process with ELECTRE and PROMETHEE. Omega, 41:820-846, 2013.

[15] S. Corrente, J.R. Figueira, and S. Greco. The SMAA-PROMETHEE method. European Journal of Operational Research, 239(2):514-522, 2014.

[16] S. Corrente, J.R. Figueira, and S. Greco. Dealing with interaction between bipolar multiple criteria preferences in PROMETHEE methods. Annals of Operations Research, 217(1):137-164, 2014.

[17] S. Corrente, J.R. Figueira, S. Greco, and R. Słowiński. A robust ranking method exending electre III to hierarchy of interacting criteria, imprecise weights and stochastic analysis. Omega, 73:1-17, 2017.

[18] M. Dağdeviren. Decision making in equipment selection: an integrated approach with AHP and PROMETHEE. Journal of Intelligent Manufacturing, 19(4):397-406, 2008.

[19] N. Doan and Y. De Smet. An alternative, weight sensitivity analysis for PROMETHEE II rankings. Omega, 2017, DOI: 10.1016/j.omega.2017.08.017.

[20] M. Goumas and V. Lygerou. An extension of the PROMETHEE method for decision making in fuzzy environment: Ranking of alternative energy exploitation projects. European Journal of Operational Research, 123(3):606-613, 2000.

[21] K. Govindan, M. Kadziński, and R. Sivakumar. Application of a novel PROMETHEE-based method for construction of a group compromise ranking to prioritization of green suppliers in food supply chain. Omega, 71:129-145, 2017.

[22] M. Grabisch and C. Labreuche. Bi-capacities-I: definition, Möbius transform and interaction. Fuzzy sets and systems, 151(2):211-236, 2005.

[23] M. Grabisch and C, Labreuche. Bi-capacities-II: the Choquet integral. Fuzzy sets and systems, 151(2):237-259, 2005.

[24] A. Greco, J.R. Figueira, and M. Ehrgott. Multiple Criteria Decision Analysis: State of the Art Surveys. Springer, Berlin, 2016.

[25] S. Greco and J.R. Figueira. Dealing with interaction between bi-polar multiple criteria preferences in outranking methods. Research Report 11-2003, INESC-Coimbra, Portugal, 2003. http://www.uc.pt/en/org/inescc/res_reports_docs/research_reports.

[26] S. Greco and F. Rindone. Bipolar fuzzy integrals. Fuzzy Sets and Systems, 220:21-33, 2013.

[27] S. Greco, B. Matarazzo, and R. Slowinski. Bipolar Sugeno and Choquet integrals. In Proceedings of the EUROFUSE 02 Workshop on Information Systems, pages 191-196, 2002. 
[28] S. Greco, V. Mousseau, and R. Słowiński. Ordinal regression revisited: multiple criteria ranking using a set of additive value functions. European Journal of Operational Research, 191(2):416436, 2008.

[29] N. Halouani, H. Chabchoub, and J.-M. Martel. PROMETHEE-MD-2T method for project selection. European Journal of Operational Research, 195(3):841-849, 2009.

[30] J.-P. Hubinont. SMAA-GAIA: a complementary tool of the SMAA-PROMETHEE method. International Journal of Multicriteria Decision Making, 6(3):237-246, 2016.

[31] K. Hyde, H.R. Maier, and C. Colby. Incorporating uncertainty in the PROMETHEE MCDA method. Journal of Multi-Criteria Decision Analysis, 12(4-5):245-259, 2003.

[32] E. Jacquet-Lagrèze and Y. Siskos. Preference disaggregation: 20 years of MCDA experience. European Journal of Operational Research, 130(2):233-245, 2001.

[33] I.T. Jolliffe. Principal Component Analysis and Factor Analysis. In Principal component analysis, pages 115-128. Springer, 1986.

[34] M. Kadziński and K. Ciomek. Integrated framework for preference modeling and robustness analysis for outranking-based multiple criteria sorting with ELECTRE and PROMETHEE. Information Sciences, 352:167-187, 2016.

[35] M. Kadziński, S. Greco, and R. Słowiński. Extreme ranking analysis in Robust Ordinal Regression. Omega, 40(4):488-501, 2012.

[36] R.L. Keeney and H. Raiffa. Decisions with multiple objectives: Preferences and value tradeoffs. J. Wiley, New York, 1976.

[37] H.S. Kilic, S. Zaim, and D. Delen. Selecting "The Best" ERP system for SMEs using a combination of ANP and PROMETHEE methods. Expert Systems with Applications, 42(5):2343-2352, 2015 .

[38] R. Lahdelma and P. Salminen. SMAA-2: Stochastic multicriteria acceptability analysis for group decision making. Operations Research, 49(3):444-454, 2001.

[39] R. Lahdelma and P. Salminen. Stochastic multicriteria acceptability analysis (SMAA). In M. Ehrgott, J. Figueira, and S. Greco, editors, Trends in Multiple Criteria Decision Analysis, pages 321-354. Springer, 2010.

[40] P. Leskinen, J. Viitanen, A. Kangas, and J. Kangas. Alternatives to incorporate uncertainty and risk attitude in/multicriteria evaluation of forest plans. Forest Science, 52(3):304-312, 2006.

[41] B. Mareschal. Stochastic multicriteria decision making and uncertainty. European Journal of Operational Research, 26(1):58-64, 1986.

[42] B. Mareschal and J.-P. Brans. Geometrical representations for MCDA. European Journal of Operational Research, 34(1):69-77, 1988.

[43] V. Mousseau, J.R. Figueira, L. Dias, C. Gomes da Silva, and J. Climaco. Resolving inconsistencies among constraints on the parameters of an MCDA model. European Journal of Operational Research, 147(1):72-93, 2003.

[44] B. Roy. Multicriteria Methodology for Decision Aiding. Nonconvex Optimization and its Applications. Kluwer Academic Publishers, Dordrecht, 1996. 
[45] R.L. Smith. Efficient Monte Carlo procedures for generating points uniformly distributed over bounded regions. Operations Research, 32:1296-1308, 1984.

[46] Z. Taha and S. Rostam. A hybrid fuzzy AHP-PROMETHEE decision support system for machine tool selection in flexible manufacturing cell. Journal of Intelligent Manufacturing, 23 (6):2137-2149, 2012.

[47] T. Tervonen and J.R. Figueira. A survey on stochastic multicriteria acceptability analysis methods. Journal of Multi-Criteria Decision Analysis, 15(1-2):1-14, 2008.

[48] T. Tervonen, G. Van Valkenhoef, N. Basturk, and D. Postmus. Efficient weight generation for simulation based multiple criteria decision analysis. EWG-MCDA, Tarragona, 12-14 April, 2012.

[49] T. Tervonen, G. Van Valkenhoef, N. Bastürk, and D. Postmus. Hit-and-run enables efficient weight generation for simulation-based multiple criteria decision analysis European Journal of Operational Research, 224:552-559, 2013.

[50] S. Wold, K. Esbensen, and P. Geladi. Principal component analysis. Chemometrics and intelligent laboratory systems, 2(1-3):37-52, 1987. 